\title{
Characterization of Oxygen-Containing Aromatics in a Low-Temperature Coal Tar
}

Hua-Shuai Gao ${ }^{\mathrm{a}}$, Zhi-Min Zong, ${ }^{\mathrm{a}}{ }^{*}$, Jia-Hao Lia, Xian-Yong Wei ${ }^{\mathrm{a}}$, b, $\dagger$, Qing-Jie Guo ${ }^{\mathrm{b}}$, Tian-Sheng Zhao $^{\text {b }}$, Hong-Cun Bai ${ }^{\mathrm{b}}$

a Key Laboratory of Coal Processing and Efficient Utilization, Ministry of Education, China University of Mining \& Technology, Xuzhou 221116, Jiangsu, China

${ }^{b}$ State Key Laboratory of High-efficiency Utilization and Green Chemical Engineering, Ningxia University, Yinchuan 750021, Ningxia, China

\section{Catalyst preparation}

Nickel carbonyl (NC) was produced by the reaction of nickel powders (NPs) with $6 \mathrm{MPa}$ of $\mathrm{CO}$ at $100{ }^{\circ} \mathrm{C}$ in a $100 \mathrm{~mL}$ stainless and magnetically stirred autoclave for $2 \mathrm{~h}$. After cooling the autoclave to room temperature, $\mathrm{CO}$ in the autoclave was released and the autoclave was pressurized with $6 \mathrm{MPa}$ of $\mathrm{CO}$ again. Then the autoclave was heated to $100{ }^{\circ} \mathrm{C}$ and maintained at $100{ }^{\circ} \mathrm{C}$ for another $2 \mathrm{~h}$ to make the complete conversion of NPs to NC. Subsequently, ethoxyethane ( $25 \mathrm{~mL})$, Z5A ( $4 \mathrm{~g})$, and NC (1 mL) were put into the autoclave. After replacing air inside the autoclave 3 times with $\mathrm{N}_{2}$, the mixture in the autoclave was stirred at $100 \mathrm{rpm}$ for $1 \mathrm{~h}$ at room temperature to fully impregnate $\mathrm{NC}$ onto $\mathrm{Z5A}$. Then the autoclave was heated to $100{ }^{\circ} \mathrm{C}$ and the mixture in the autoclave was stirred at $250 \mathrm{rpm}$ for $1 \mathrm{~h}$. After cooling the autoclave to room temperature, $\mathrm{CO}$ in the autoclave was released followed by repeating the operation steps as mentioned above to decompose $\mathrm{NC}$ as completely as possible. Finally, the reaction mixture was removed from the autoclave and filtrated to obtain $\mathrm{Ni} / \mathrm{Z} 5 \mathrm{~A}$ after desiccation in a vacuum at $60^{\circ} \mathrm{C}$ for $4 \mathrm{~h}$.

\footnotetext{
${ }^{*}$ Corresponding author. Tel: +8651683885951 ; fax: +86 51683884399.

E-mail address: zong_zhimin@163.com (Z. M. Zong).

$\dagger$ Corresponding author. Tel: +86516 83885951; fax: +8651683884399.

E-mail address:wei_xianyong@163.com (X. Y. Wei).
} 

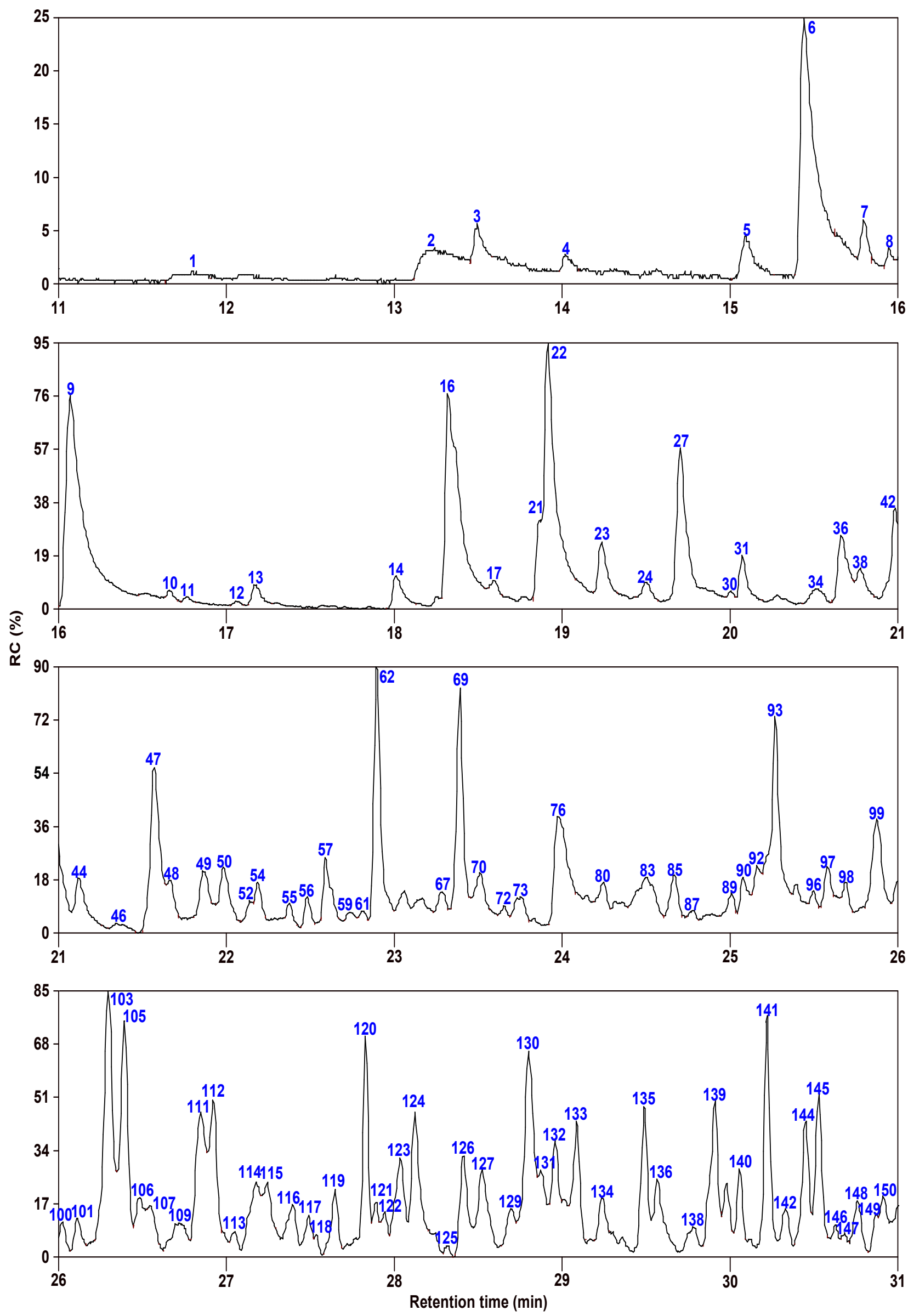

Figure S1. Total ion chromatogram of the LTCT. 

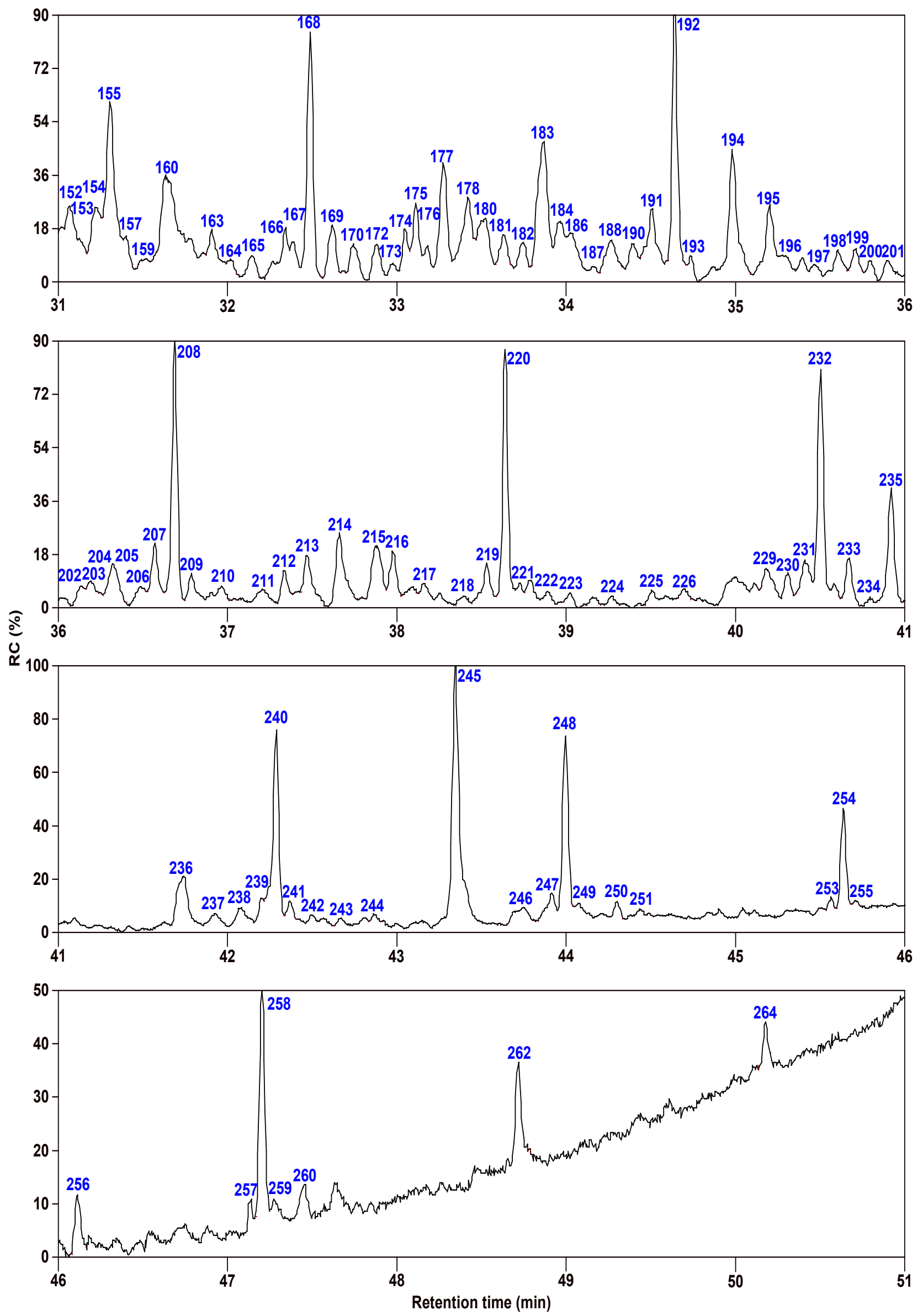

Figure S1. Total ion chromatogram of the LTCT (continued). 

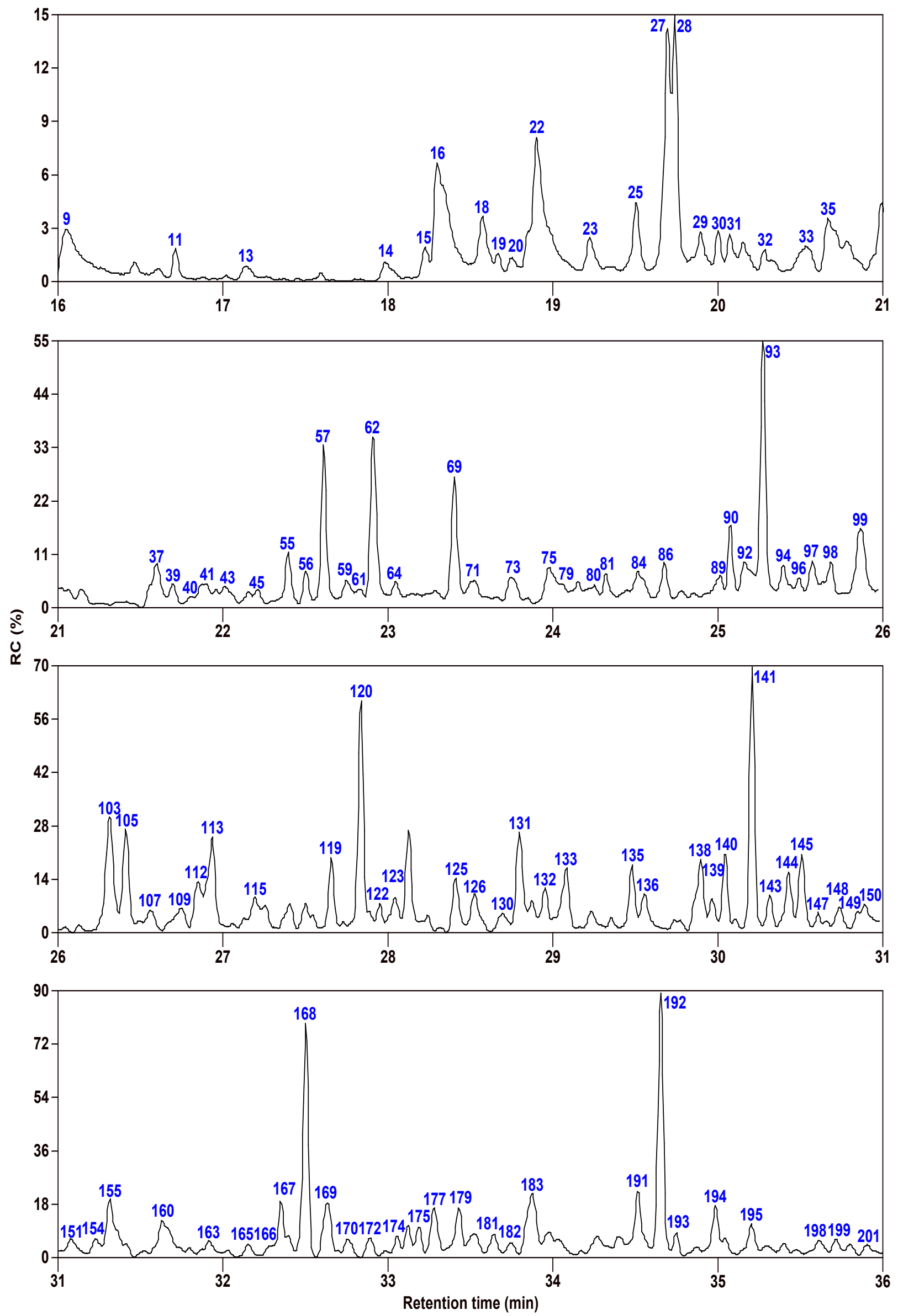

Figure S2. Total ion chromatogram of $\mathrm{UP}_{2}$. 

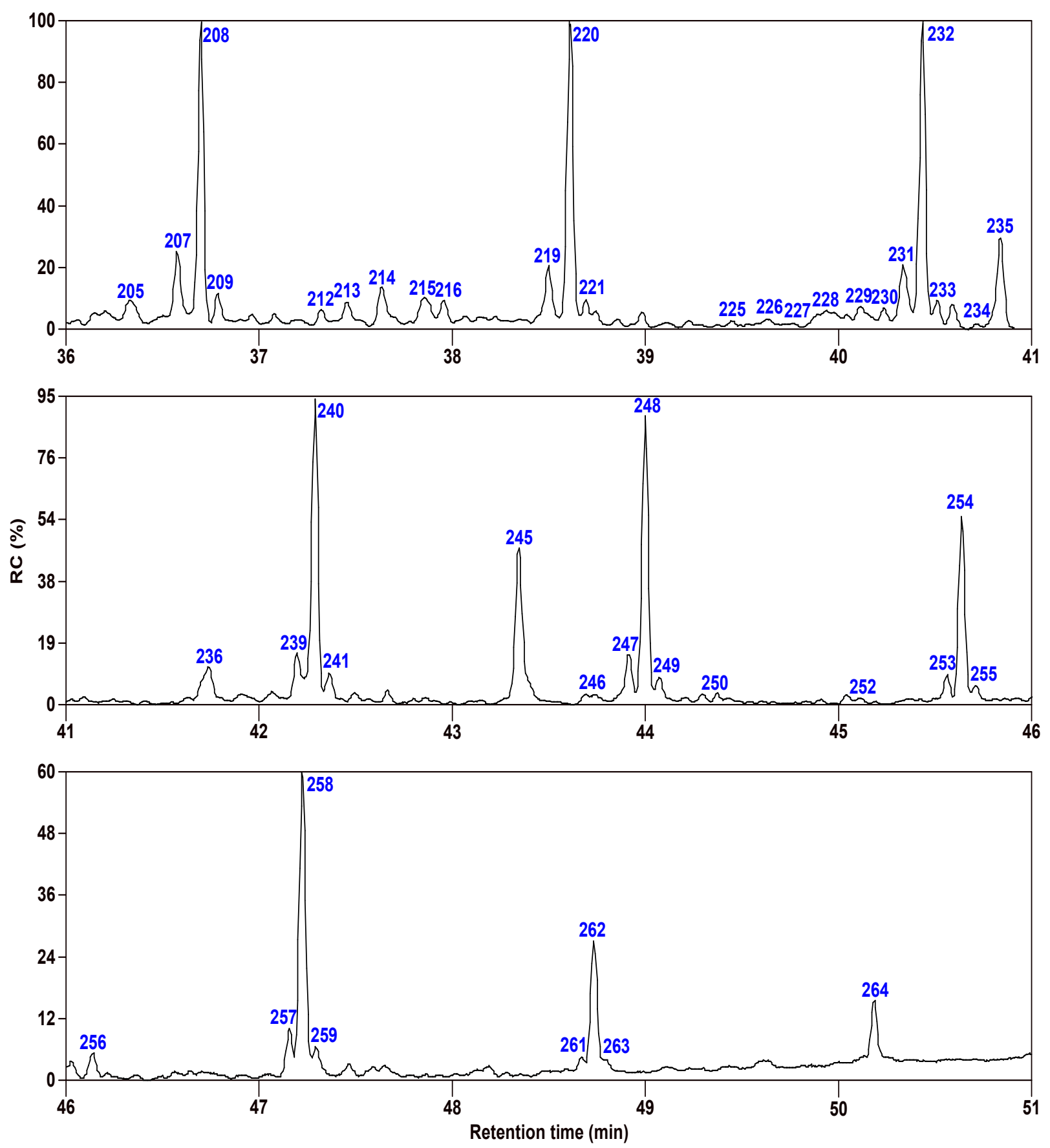

Figure S2. Total ion chromatogram of $\mathrm{UP}_{2}$ (continued). 

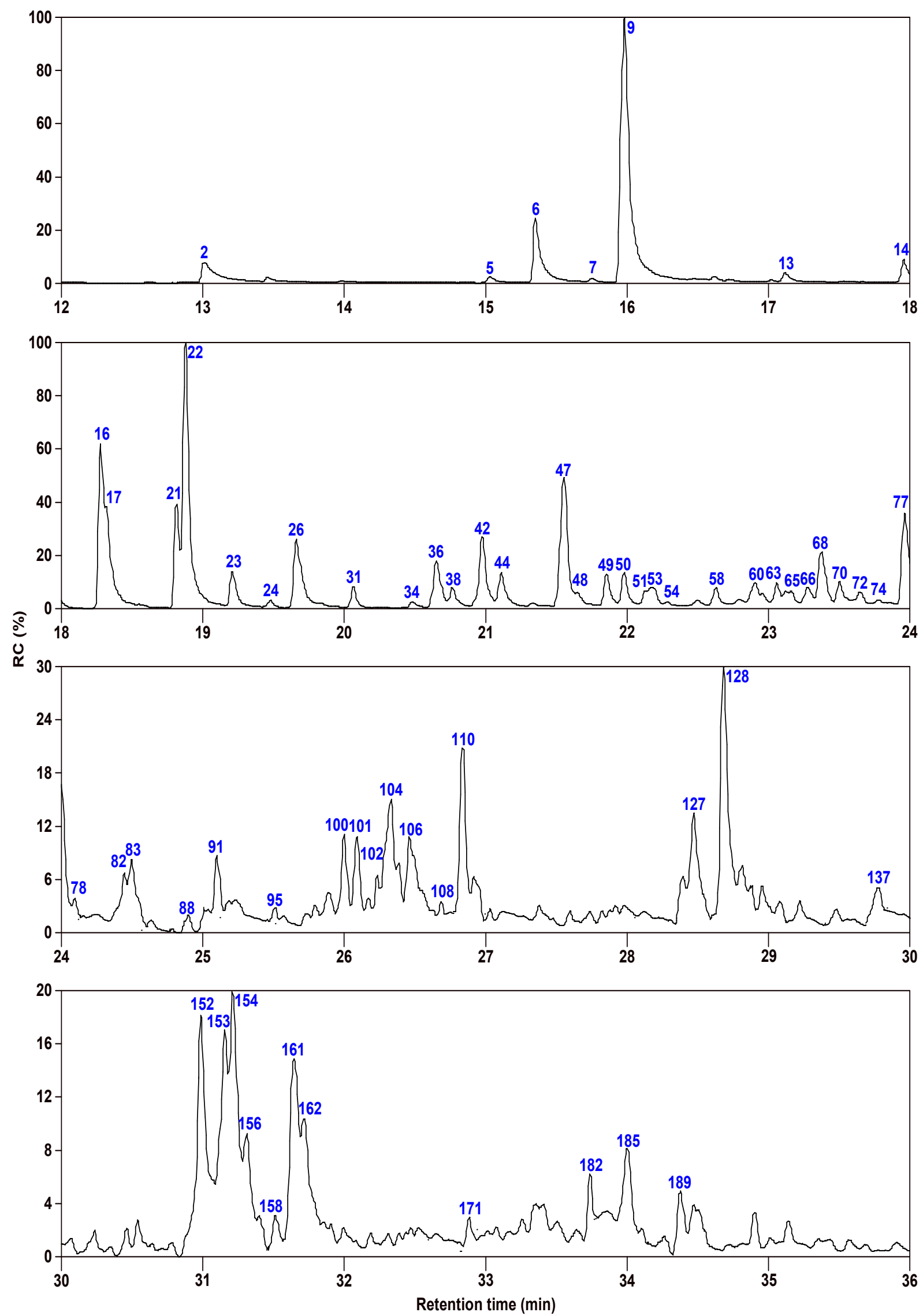

Figure S3. Total ion chromatogram of $\mathrm{LP}_{2}$. 

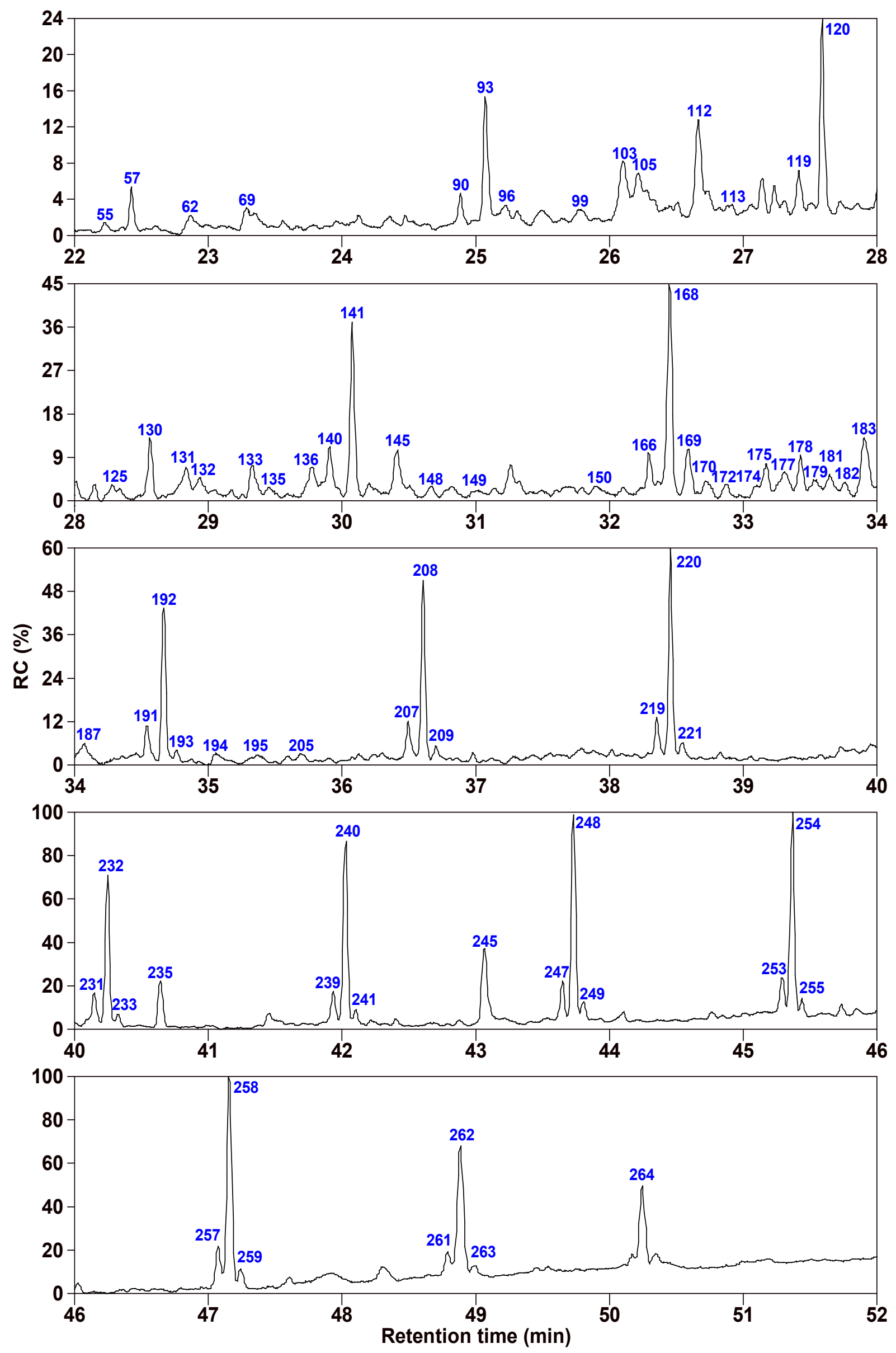

Figure S4. Total ion chromatogram of $\mathrm{HCUP}_{2}$. 
Table S1. NAs Detected in LTCT, $\mathrm{UP}_{2}$, and $\mathrm{HCUP}_{2}$.

\begin{tabular}{|c|c|c|c|c|c|c|c|c|c|}
\hline \multirow{2}{*}{ peak } & \multirow{2}{*}{ NA } & \multicolumn{3}{|l|}{$\mathrm{RC}$} & \multirow{2}{*}{ peak } & \multirow{2}{*}{ NA } & \multicolumn{3}{|l|}{$\mathrm{RC}$} \\
\hline & & LTCT & $\mathrm{UP}_{2}$ & $\mathrm{HCUP}_{2}$ & & & LTCT & $\mathrm{UP}_{2}$ & $\mathrm{HCUP}_{2}$ \\
\hline 28 & dodecane & & 0.32 & & 220 & icosane & 1.69 & 4.53 & 5.02 \\
\hline 57 & tridecane & 0.70 & 1.49 & 0.48 & 232 & henicosane & 1.54 & 4.28 & 5.67 \\
\hline 93 & tetradecane & 1.61 & 2.33 & 1.40 & 240 & docosane & 1.47 & 4.08 & 7.23 \\
\hline 120 & pentadecane & 1.23 & 2.53 & 1.98 & 248 & tricosane & 1.36 & 3.90 & 7.95 \\
\hline 141 & hexadecane & 1.57 & 3.26 & 2.74 & 254 & tetracosane & 0.74 & 2.53 & 7.61 \\
\hline 168 & heptadecane & 1.85 & 3.81 & 3.27 & 258 & pentacosane & 0.58 & 1.81 & 7.22 \\
\hline 192 & octadecane & 1.83 & 4.08 & 3.65 & 262 & hexacosane & 0.31 & 0.77 & 4.32 \\
\hline 208 & nonadecane & 1.85 & 4.40 & 4.39 & 264 & heptacosane & 0.13 & 0.36 & 2.55 \\
\hline
\end{tabular}

Table S2. BAs ${ }^{\mathrm{I}}$ Detected in LTCT, $\mathrm{UP}_{2}$, and HCUP ${ }_{2}$.

\begin{tabular}{llllllll} 
peak & \multirow{2}{*}{$\mathrm{BA}^{\mathrm{I}}$} & \multirow{2}{*}{$\mathrm{RC}$ in $\mathrm{UP}_{2}$} & peak & \multirow{2}{*}{$\mathrm{BA}^{\mathrm{I}}$} & \multicolumn{2}{l}{$\mathrm{RC}$} & \\
\cline { 5 - 7 } & & & & $\mathrm{LTCT}$ & $\mathrm{UP}_{2}$ & $\mathrm{HCUP}_{2}$ \\
\hline $\mathbf{3 2}$ & 2-methylundecane & 0.04 & $\mathbf{8 6}$ & 4,6-dimethylundecane & & 0.43 & \\
$\mathbf{8 1}$ & 2,-dimethylundecane & 0.03 & $\mathbf{1 6 9}$ & pristane & 0.51 & 1.21 & 0.92 \\
\hline
\end{tabular}

Table S3. ASCHs Detected in LTCT and $\mathrm{UP}_{2}$.

\begin{tabular}{lllllll}
\multirow{2}{*}{ peak } & \multirow{2}{*}{ ASCH } & RC & \multirow{2}{*}{ peak } & ASCH & RC & in \\
\cline { 3 - 5 } & & LTCT & $\mathrm{UP}_{2}$ & & & LTCT \\
\hline $\mathbf{1 2}$ & ethylcyclohexane & 0.04 & & $\mathbf{1 7 6}$ & 2-butyl-1,1,4-trimethylcyclohexane & 0.08 \\
$\mathbf{7 9}$ & isopropylcyclohexane & & 0.07 & & & \\
\hline
\end{tabular}

Table S4. Alk-1-enes Detected in LTCT, UP $\mathbf{P}_{2}$, and HCUP $\mathbf{P}_{2}$.

\begin{tabular}{|c|c|c|c|c|c|c|c|c|c|}
\hline \multirow{2}{*}{ peak } & \multirow{2}{*}{ alk-1-ene } & \multicolumn{3}{|l|}{$\mathrm{RC}$} & \multirow{2}{*}{ peak } & \multirow{2}{*}{ alk-1-ene } & \multicolumn{3}{|l|}{$\mathrm{RC}$} \\
\hline & & LTCT & $\mathrm{UP}_{2}$ & $\mathrm{HCUP}_{2}$ & & & LTCT & $\mathrm{UP}_{2}$ & $\mathrm{HCUP}_{2}$ \\
\hline 25 & dodec-1-ene & & 0.28 & & 219 & icos-1-ene & 0.18 & 0.98 & 0.79 \\
\hline 55 & tridec-1-ene & 0.16 & 0.47 & 0.11 & 231 & henicos-1-ene & 0.24 & 0.89 & 1.15 \\
\hline 90 & tetradec-1-ene & 0.23 & 0.55 & 0.36 & 239 & docos-1-ene & 0.08 & 0.42 & 1.16 \\
\hline 119 & pentadec-1-ene & 0.45 & 0.81 & 0.52 & 247 & tricos-1-ene & 0.24 & 0.61 & 1.35 \\
\hline 140 & hexadec-1-ene & 0.40 & 0.79 & 0.55 & 253 & tetracos-1-ene & 0.07 & 0.28 & 1.12 \\
\hline 166 & heptadec-1-ene & 0.13 & 0.49 & 0.45 & 257 & pentacos-1-ene & 0.04 & 0.17 & 1.02 \\
\hline 191 & octadec-1-ene & 0.23 & 1.07 & 0.92 & 261 & hexacos-1-ene & & 0.05 & 0.41 \\
\hline 207 & nonadec-1-ene & 0.34 & 0.96 & 0.93 & & & & & \\
\hline
\end{tabular}

Table S5. Alk-2-enes Detected in LTCT, $\mathrm{UP}_{2}$, and HCUP $\mathrm{P}_{2}$.

\begin{tabular}{|c|c|c|c|c|c|c|c|c|c|}
\hline \multirow{2}{*}{ peak } & \multirow{2}{*}{ alk-2-ene } & \multicolumn{3}{|l|}{$\mathrm{RC}$} & \multirow[b]{2}{*}{ peak } & \multirow{2}{*}{ alk-2-ene } & \multicolumn{3}{|l|}{$\mathrm{RC}$} \\
\hline & & LTCT & $\mathrm{UP}_{2}$ & $\mathrm{HCUP}_{2}$ & & & LTCT & $\mathrm{UP}_{2}$ & $\mathrm{HCUP}_{2}$ \\
\hline 29 & dodec-2-ene & & 0.11 & & 221 & icos-2-ene & 0.04 & 0.17 & 0.31 \\
\hline 59 & tridec-2-ene & 0.05 & 0.15 & & 233 & henicos-2-ene & 0.05 & 0.22 & 0.35 \\
\hline 94 & tetradec-2-ene & & 0.18 & & 241 & docos-2-ene & 0.13 & 0.26 & 0.37 \\
\hline 122 & pentadec-2-ene & 0.09 & 0.15 & & 249 & tricos-2-ene & 0.05 & 0.20 & 0.53 \\
\hline 143 & hexadec-2-ene & & 0.39 & & 255 & tetracos-2-ene & 0.03 & 0.11 & 0.44 \\
\hline 193 & octadec-2-ene & 0.11 & 0.28 & 0.27 & 259 & pentacos-2-ene & 0.02 & 0.08 & 0.43 \\
\hline 209 & nonadec-2-ene & 0.16 & 0.38 & 0.32 & 263 & hexacos-2-ene & & 0.02 & 0.21 \\
\hline
\end{tabular}

Table S6. NSAs ${ }^{\mathrm{I}}$ Detected in LTCT, $\mathrm{UP}_{2}$, and HCUP . $_{\text {. }}$

\begin{tabular}{|c|c|c|c|c|c|c|c|c|}
\hline \multirow{2}{*}{ peak } & \multirow{2}{*}{$\mathrm{NSA}^{\mathrm{I}}$} & \multicolumn{3}{|l|}{$\mathrm{RC}$} & \multirow{2}{*}{ peak } & \multirow{2}{*}{$\mathrm{NSA}^{\mathrm{I}}$} & \multicolumn{2}{|l|}{$\mathrm{RC}$} \\
\hline & & LTCT & $\mathrm{UP}_{2}$ & $\mathrm{HCUP}_{2}$ & & & LTCT & $\mathrm{UP}_{2}$ \\
\hline 27 & naphthalene & 2.31 & 0.30 & & 234 & fluoranthene & 0.40 & 0.35 \\
\hline 114 & acenaphthene & 0.30 & & & 244 & pyrene & 0.06 & \\
\hline 194 & phenanthrene & 1.26 & 0.63 & 0.39 & 260 & tetraphene & 0.12 & \\
\hline 195 & anthracene & 0.52 & 0.46 & 0.58 & & & & \\
\hline
\end{tabular}


Table S7. BAs ${ }^{\mathrm{II}}$ Detected in LTCT and $\mathrm{UP}_{2}$.

\begin{tabular}{lllllll}
\hline \multirow{2}{*}{ peak } & \multirow{2}{*}{ BA $^{\mathrm{II}}$} & \multicolumn{2}{l}{ RC } & \multirow{2}{*}{ peak } & \multirow{2}{*}{ BA } & \multirow{2}{*}{ RC in LTCT } \\
\cline { 3 - 6 } & LTCT & $\mathrm{UP}_{2}$ & & \\
\hline $\mathbf{9 2}$ & biphenyl & 0.11 & 0.28 & $\mathbf{2 2 2}$ & 2-phenylnaphthalene & 0.12 \\
\hline
\end{tabular}

Table S8. SMCNSAs Detected in LTCT and $\mathrm{UP}_{2}$.

\begin{tabular}{lllllll}
\hline \multirow{2}{*}{ peak } & \multirow{2}{*}{ SMCNSA } & \multicolumn{2}{l}{ RC } & \multirow{2}{*}{ peak } & \multirow{2}{*}{ SMCNSA } & \multirow{2}{*}{ RC in LTCT } \\
\cline { 3 - 5 } & & LTCT & $\mathrm{UP}_{2}$ & & \\
\hline $\mathbf{1 4 4}$ & fluorene & 0.74 & 0.63 & $\mathbf{2 5 1}$ & benzo[b]fluorene & 0.06 \\
\hline
\end{tabular}

Table S9. ASBs Detected in LTCT, $\mathrm{UP}_{2}$, and HCUP $\mathbf{P}_{2}$.

\begin{tabular}{|c|c|c|c|c|c|c|c|c|}
\hline \multirow{2}{*}{ peak } & \multirow{2}{*}{ ASB } & \multicolumn{2}{|l|}{$\mathrm{RC}$} & \multirow{2}{*}{ peak } & \multirow{2}{*}{ ASB } & \multicolumn{3}{|l|}{$\mathrm{RC}$} \\
\hline & & LTCT & $\mathrm{UP}_{2}$ & & & LTCT & $\mathrm{UP}_{2}$ & $\mathrm{HCUP}_{2}$ \\
\hline 15 & sec-butylbenzene & & 0.06 & 113 & hexamethylbenzene & 0.10 & 1.14 & 0.07 \\
\hline 84 & heptylbenzene & & 0.37 & 117 & 1,3,5-triethylbenzene & 0.16 & & \\
\hline 85 & 3,4-dimethylcumene & 0.49 & & 146 & ethylpentamethylbenzene & 0.06 & & \\
\hline
\end{tabular}

Table S10. ASDPMs Detected in LTCT and HCUP 2 .

\begin{tabular}{lllllll}
\hline \multirow{2}{*}{ peak } & \multirow{2}{*}{ ASDPM } & RC & & \multirow{2}{*}{ peak } & \multirow{2}{*}{ ASDPM } & \multirow{2}{*}{ RC in LTCT } \\
\cline { 3 - 5 } & & LTCT & $\mathrm{HCUP}_{2}$ & & & \\
\hline $\mathbf{1 8 7}$ & di- $m$-tolylmethane & 0.05 & 0.40 & $\mathbf{2 1 1}$ & di- $p$-tolylmethane & 0.19 \\
$\mathbf{2 0 3}$ & 1-benzyl-4-ethylbenzene & 0.07 & & & & \\
\hline
\end{tabular}

Table S11. MIs Detected in LTCT.

\begin{tabular}{llllll}
\hline peak & MI & RC & peak & MI & RC \\
\hline $\mathbf{2 0}$ & 2-MI & 0.06 & $\mathbf{3 7}$ & 4-MI & 0.17 \\
\hline
\end{tabular}

Table S12. DMIs ${ }^{I}$ Detected in LTCT and $\mathrm{UP}_{2}$.

\begin{tabular}{|c|c|c|c|c|c|c|c|c|c|}
\hline \multirow{2}{*}{ peak } & \multirow{2}{*}{$\mathrm{DMI}^{\mathrm{I}}$} & \multirow{2}{*}{$\mathrm{RC}$ in $\mathrm{UP}_{2}$} & \multirow{2}{*}{ peak } & \multirow{2}{*}{$\mathrm{DMI}^{\mathrm{I}}$} & \multirow{2}{*}{$\mathrm{RC}$ in $\mathrm{UP}_{2}$} & \multirow{2}{*}{ peak } & \multirow{2}{*}{$\mathrm{DMI}^{\mathrm{I}}$} & \multicolumn{2}{|l|}{$\mathrm{RC}$} \\
\hline & & & & & & & & LTCT & $\mathrm{UP}_{2}$ \\
\hline 39 & 4,7-DMI & 0.03 & 43 & $2,3-\mathrm{DMI}^{\mathrm{I}}$ & 0.13 & 52 & $3,5-\mathrm{DMI}^{\mathrm{I}}$ & 0.05 & \\
\hline 40 & $1,3-\mathrm{DMI}^{\mathrm{I}}$ & 0.04 & 45 & $1,1-\mathrm{DMI}^{\mathrm{I}}$ & 0.08 & 56 & 4,6-DMI & 0.21 & 0.31 \\
\hline
\end{tabular}

Table S13. TMIs ${ }^{\mathrm{I}}$ Detected in LTCT and $\mathrm{UP}_{2}$.

\begin{tabular}{lllllll}
\hline peak & \multirow{2}{*}{$\mathrm{TMI}^{\mathrm{I}}$} & \multirow{2}{*}{$\mathrm{RC}_{\text {in } \mathrm{UP}_{2}}$} & peak & \multirow{2}{*}{$\mathrm{TMI}^{\mathrm{I}}$} & \multicolumn{2}{l}{ RC } \\
\cline { 4 - 7 } & & & & LTCT & $\mathrm{UP}_{2}$ \\
\hline $\mathbf{8 7}$ & $1,1,3-\mathrm{TMI}^{\mathrm{I}}$ & 0.07 & $\mathbf{8 9}$ & $1,2,3-\mathrm{TMI}^{\mathrm{I}}$ & 0.22 & 0.17 \\
\hline
\end{tabular}

Table S14. MSIs Detected in LTCT, $\mathrm{UP}_{2}$, and HCUP

\begin{tabular}{lllllllll}
\hline \multirow{2}{*}{ peak } & \multirow{2}{*}{ MSI } & \multicolumn{2}{l}{ RC } & & \multirow{2}{*}{ peak } & MSI & RC & \\
\cline { 3 - 7 } & & LTCT & $\mathrm{UP}_{2}$ & & & LTCT & $\mathrm{UP}_{2}$ & $\mathrm{HCUP}_{2}$ \\
\hline $\mathbf{1 8}$ & 1-methylindane & & 0.19 & $\mathbf{7 3}$ & 4,5,7-trimethylindane & 0.31 & 0.42 & \\
$\mathbf{3 0}$ & 1,2-dimethylindane & 0.05 & 0.11 & $\mathbf{9 6}$ & 1,1,3-trimethylindane & 0.11 & 0.10 & 0.07 \\
$\mathbf{4 1}$ & 1,6-dimethylindane & & 0.03 & $\mathbf{1 0 7}$ & 1,1,5-trimethylindane & 0.14 & 0.27 & \\
$\mathbf{7 1}$ & 1,1,2-trimethylindane & & 0.23 & $\mathbf{1 3 0}$ & 1,1,4,5,6-pentamethylindane & 1.22 & 1.19 & 0.95 \\
\hline
\end{tabular}

Table S15. MBPs Detected in LTCT and $\mathrm{UP}_{2}$.

\begin{tabular}{|c|c|c|c|c|c|c|}
\hline \multirow{2}{*}{ peak } & \multirow{2}{*}{ MBP } & \multirow{2}{*}{$\mathrm{RC}$ in $\mathrm{LTCT}$} & \multirow{2}{*}{ peak } & \multirow{2}{*}{ MBP } & $\mathrm{RC}$ & \\
\hline & & & & & LTCT & $\mathrm{UP}_{2}$ \\
\hline 121 & 1-MBP & 0.07 & 147 & 4-MBP & 0.03 & 0.04 \\
\hline
\end{tabular}

Table S16. DMBPs Detected in LTCT, $\mathrm{UP}_{2}$ and HCUP $\mathrm{P}_{2}$.

\begin{tabular}{|c|c|c|c|c|c|c|c|c|}
\hline \multirow{2}{*}{ peak } & \multirow{2}{*}{ DMBP } & \multicolumn{3}{|l|}{$\mathrm{RC}$} & \multirow{2}{*}{ peak } & \multirow{2}{*}{ DMBP } & \multicolumn{2}{|l|}{$\mathrm{RC}$} \\
\hline & & LTCT & $\mathrm{UP}_{2}$ & $\mathrm{HCUP}_{2}$ & & & $\mathrm{UP}_{2}$ & $\mathrm{HCUP}_{2}$ \\
\hline 150 & 2,2'-DMBP & 0.11 & 0.10 & 0.13 & 179 & 4,4'-DMBP & 0.38 & 0.20 \\
\hline 165 & 2,3-DMBP & 0.24 & 0.25 & & & & & \\
\hline
\end{tabular}


Table S17. TMBPs Detected in LTCT, $\mathrm{UP}_{2}$, and HCUP $\mathrm{H}_{2}$.

\begin{tabular}{llllllll}
\hline \multirow{2}{*}{ peak } & \multirow{2}{*}{ TMBP } & \multicolumn{2}{l}{ RC } & \multirow{2}{*}{ peak } & \multirow{2}{*}{ TMBP } & \multirow{2}{*}{ RC in LTCT } \\
\cline { 3 - 6 } & & LTCT & $\mathrm{UP}_{2}$ & $\mathrm{HCUP}_{2}$ & & & \multirow{2}{*}{$\mathbf{2 0 6}$} \\
$\mathbf{1 9 9}$ & $2,2^{\prime}, 5,5^{\prime}-\mathrm{T}$-TMBP & 0.15 & 0.23 & & $4^{\prime}-\mathrm{TMBP}$ & 0.12 \\
$\mathbf{2 0 5}$ & $2,2^{\prime}, 6,66^{\prime}-\mathrm{TMBP}$ & 0.49 & 0.52 & 0.20 & & & \\
\hline
\end{tabular}

Table S18. MFs Detected in LTCT, $\mathrm{UP}_{2}$ and HCUP $\mathrm{P}_{2}$.

\begin{tabular}{|c|c|c|c|c|c|c|c|c|c|}
\hline \multirow{2}{*}{ peak } & \multirow{2}{*}{ MF } & \multicolumn{3}{|l|}{$\mathrm{RC}$} & \multirow{2}{*}{ peak } & \multirow{2}{*}{ MF } & \multicolumn{3}{|l|}{$\mathrm{RC}$} \\
\hline & & LTCT & $\mathrm{UP}_{2}$ & $\mathrm{HCUP}_{2}$ & & & LTCT & $\mathrm{UP}_{2}$ & $\mathrm{HCUP}_{2}$ \\
\hline 174 & 1-MF & 0.17 & 0.21 & 0.10 & 177 & 4-MF & 0.93 & 0.88 & 0.60 \\
\hline 175 & 2-MF & 0.31 & 0.27 & 0.45 & 180 & 9-MF & 0.36 & & \\
\hline
\end{tabular}

Table S19. DMFs Detected in LTCT and $\mathrm{UP}_{2}$.

\begin{tabular}{|c|c|c|c|c|c|c|c|c|c|c|}
\hline \multirow{2}{*}{ peak } & \multirow{2}{*}{$\mathrm{DMF}$} & \multicolumn{2}{|l|}{$\mathrm{RC}$} & \multirow{2}{*}{ peak } & \multirow{2}{*}{ DMF } & \multirow{2}{*}{$\mathrm{RC}$ in $\mathrm{LTCT}$} & \multirow{2}{*}{ peak } & \multirow{2}{*}{ DMF } & \multicolumn{2}{|l|}{$\mathrm{RC}$} \\
\hline & & LTCT & $\mathrm{UP}_{2}$ & & & & & & LTCT & $\mathrm{UP}_{2}$ \\
\hline 198 & 2,3-DMF & 0.19 & 0.22 & 200 & 1,7-DMF & 0.12 & 201 & 9,9-DMF & 0.16 & 0.17 \\
\hline
\end{tabular}

Table S20. MNs ${ }^{\mathrm{I}}$ Detected in LTCT, UP ${ }_{2}$, and HCUP $\mathrm{H}_{2}$.

\begin{tabular}{|c|c|c|c|c|c|c|c|c|c|}
\hline \multirow{2}{*}{ peak } & \multirow{2}{*}{$\mathrm{MN}^{\mathrm{I}}$} & \multicolumn{3}{|l|}{$\mathrm{RC}$} & \multirow{2}{*}{ peak } & \multirow{2}{*}{$\mathrm{MN}^{\mathrm{I}}$} & \multicolumn{3}{|l|}{$\mathrm{RC}$} \\
\hline & & LTCT & $\mathrm{UP}_{2}$ & $\mathrm{HCUP}_{2}$ & & & LTCT & $\mathrm{UP}_{2}$ & $\mathrm{HCUP}_{2}$ \\
\hline 62 & $2-\mathrm{MN}^{\mathrm{I}}$ & 2.53 & 1.90 & 0.27 & 69 & 1-MN & 2.11 & 1.38 & 0.10 \\
\hline
\end{tabular}

Table S21. ENs Detected in LTCT and $\mathrm{UP}_{2}$.

\begin{tabular}{lllllllll}
\hline \multirow{2}{*}{ peak } & \multirow{2}{*}{ EN } & \multicolumn{2}{l}{ RC } & \multicolumn{2}{l}{ peak } & \multirow{2}{*}{ EN } & \multicolumn{2}{l}{ RC } \\
\cline { 3 - 7 } & & LTCT & $\mathrm{UP}_{2}$ & & LTCT & $\mathrm{UP}_{2}$ \\
\hline $\mathbf{9 7}$ & 2-EN & 0.37 & 0.25 & $\mathbf{9 8}$ & $1-\mathrm{EN}$ & 0.20 & 0.26 \\
\hline
\end{tabular}

Table S22. IPNs Detected in LTCT, $\mathrm{UP}_{2}$, and HCUP ${ }_{2}$.

\begin{tabular}{|c|c|c|c|c|c|c|c|c|c|}
\hline \multirow{2}{*}{ peak } & \multirow{2}{*}{ IPN } & \multicolumn{3}{|l|}{$\mathrm{RC}$} & \multirow{2}{*}{ peak } & \multirow{2}{*}{ IPN } & \multicolumn{3}{|l|}{$\mathrm{RC}$} \\
\hline & & LTCT & $\mathrm{UP}_{2}$ & $\mathrm{HCUP}_{2}$ & & & LTCT & $\mathrm{UP}_{2}$ & $\mathrm{HCUP}_{2}$ \\
\hline 148 & 2-IPN & 0.40 & 0.34 & 0.19 & 149 & 1-IPN & 0.06 & 0.07 & 0.11 \\
\hline
\end{tabular}

Table S23. DANs Detected in LTCT, $\mathrm{UP}_{2}$, and HCUP $P_{2}$.

\begin{tabular}{lllll}
\multirow{2}{*}{ peak } & \multirow{2}{*}{ DAN } & $\mathrm{RC}$ & & \\
\cline { 3 - 5 } & & $\mathrm{LTCT}$ & $\mathrm{UP}_{2}$ & $\mathrm{HCUP}_{2}$ \\
$\mathbf{9 9}$ & 1,5-dimethylnaphthalene & 1.28 & 1.08 & 0.14 \\
$\mathbf{1 0 3}$ & 2,6-dimethylnaphthalene & 1.80 & 1.75 & 0.77 \\
$\mathbf{1 0 5}$ & 1,7-dimethylnaphthalene & 1.16 & 1.18 & \\
$\mathbf{1 1 1}$ & 1,3-dimethylnaphthalene & 0.77 & & 0.21 \\
$\mathbf{1 1 2}$ & 1,4-dimethylnaphthalene & 0.46 & 0.31 & 1.07 \\
$\mathbf{1 1 5}$ & 2,3-dimethylnaphthalene & 0.17 & 0.14 & \\
$\mathbf{1 6 7}$ & 7-isopropyl-1-methylnaphthalene & 0.07 & 0.07 & \\
$\mathbf{1 7 0}$ & 4-isopropyl-1-methylnaphthalene & 0.33 & 0.34 & 0.35 \\
$\mathbf{2 3 5}$ & 7-butyl-1-hexylnaphthalene & 0.96 & 1.56 & 2.02 \\
\hline
\end{tabular}

Table S24. TMNs ${ }^{\mathrm{I}}$ Detected in LTCT, $\mathrm{UP}_{2}$, and HCUP ${ }_{2}$.

\begin{tabular}{|c|c|c|c|c|c|c|c|c|c|}
\hline \multirow{2}{*}{ peak } & \multirow{2}{*}{$\mathrm{TMN}^{\mathrm{I}}$} & \multicolumn{3}{|l|}{$\mathrm{RC}$} & \multirow{2}{*}{ peak } & \multirow{2}{*}{$\mathrm{TMN}^{\mathrm{I}}$} & \multicolumn{3}{|l|}{$\mathrm{RC}$} \\
\hline & & LTCT & $\mathrm{UP}_{2}$ & $\mathrm{HCUP}_{2}$ & & & LTCT & $\mathrm{UP}_{2}$ & $\mathrm{HCUP}_{2}$ \\
\hline 125 & $2,3,6-\mathrm{TMN}^{\mathrm{I}}$ & 0.06 & 0.73 & 0.07 & 135 & $1,2,7-\mathrm{TMN}^{\mathrm{I}}$ & 0.89 & 0.67 & 0.14 \\
\hline 126 & $1,4,6-\mathrm{TMN}^{\mathrm{I}}$ & 0.67 & 0.28 & & 136 & $1,2,4-\mathrm{TMN}^{\mathrm{I}}$ & 0.31 & 0.34 & 0.51 \\
\hline 129 & $1,3,7-\mathrm{TMN}^{\mathrm{I}}$ & 0.19 & & & 138 & $1,2,6-\mathrm{TMN}^{\mathrm{I}}$ & 1.12 & 1.08 & \\
\hline 131 & $1,3,5-\mathrm{TMN}^{\mathrm{I}}$ & 0.08 & 0.13 & 0.54 & 139 & $1,3,8-\mathrm{TMN}^{\mathrm{I}}$ & 0.26 & 0.21 & \\
\hline 132 & $1,3,6-\mathrm{TMN}^{\mathrm{I}}$ & 0.39 & 0.36 & 0.32 & 142 & $1,4,5-\mathrm{TMN}^{\mathrm{I}}$ & 0.25 & & \\
\hline 133 & $1,6,7-\mathrm{TMN}^{\mathrm{I}}$ & 0.78 & 0.88 & 0.68 & 145 & $1,2,3-\mathrm{TMN}^{\mathrm{I}}$ & 0.91 & 0.89 & 0.83 \\
\hline
\end{tabular}


Table S25. IPDMNs Detected in LTCT, $\mathrm{UP}_{2}$, and HCUP $\mathrm{H}_{2}$.

\begin{tabular}{|c|c|c|c|c|c|c|c|}
\hline \multirow{2}{*}{ peak } & \multirow{2}{*}{ IPDMN } & \multicolumn{3}{|l|}{$\mathrm{RC}$} & \multirow{2}{*}{ peak } & \multirow{2}{*}{ IPDMN } & \multirow{2}{*}{$\mathrm{RC}$ in LTCT } \\
\hline & & LTCT & $\mathrm{UP}_{2}$ & $\mathrm{HCUP}_{2}$ & & & \\
\hline 181 & 4-IPDMN & 0.29 & 0.37 & 0.29 & 210 & 1-IPDMN & 0.09 \\
\hline
\end{tabular}

Table S26. TMNs ${ }^{\text {II }}$ Detected in LTCT, $\mathrm{UP}_{2}$, and HCUP ${ }_{2}$.

\begin{tabular}{llllllll}
\hline \multirow{2}{*}{ peak } & \multirow{2}{*}{ TMN $^{\mathrm{II}}$} & \multirow{2}{*}{ RC in LTCT } & peak & \multirow{2}{*}{$\mathrm{TMN}^{\mathrm{II}}$} & \multicolumn{2}{l}{$\mathrm{RC}$} & \\
\cline { 5 - 7 } & & & & & LTCT & $\mathrm{UP}_{2}$ & $\mathrm{HCUP}_{2}$ \\
\hline $\mathbf{1 5 7}$ & $1,2,3,4-\mathrm{TMN}^{\mathrm{II}}$ & 0.07 & $\mathbf{1 8 3}$ & $1,4,5,8-\mathrm{TMN}^{\mathrm{II}}$ & 1.44 & 1.44 & 1.34 \\
\hline
\end{tabular}

Table S27. MPs Detected in LTCT and UP 2 .

\begin{tabular}{|c|c|c|c|c|c|c|c|c|c|c|c|}
\hline \multirow{2}{*}{ peak } & \multirow{2}{*}{ MP } & \multicolumn{2}{|l|}{$\mathrm{RC}$} & \multirow{2}{*}{ peak } & \multirow{2}{*}{ MP } & \multicolumn{2}{|l|}{$\mathrm{RC}$} & \multirow{2}{*}{ peak } & \multirow{2}{*}{$\mathrm{Ml}$} & \multicolumn{2}{|l|}{$\mathrm{RC}$} \\
\hline & & LTCT & $\mathrm{UP}_{2}$ & & & LTCT & $\mathrm{UP}_{2}$ & & & LTCT & $\mathrm{UP}_{2}$ \\
\hline 212 & 2-MP & 0.24 & 0.23 & 213 & 1-MP & 0.33 & 0.32 & 215 & 4-MP & 0.52 & 0.54 \\
\hline
\end{tabular}

Table S28. DAPs Detected in LTCT, $\mathrm{UP}_{2}$, and $\mathrm{HCUP}_{2}$.

\begin{tabular}{lllll}
\hline \multirow{2}{*}{ peak } & \multirow{2}{*}{ DAP } & \multicolumn{2}{l}{$\mathrm{RC}$} & \\
\cline { 3 - 4 } & & $\mathrm{LTCT}$ & $\mathrm{UP}_{2}$ & $\mathrm{HCUP}_{2}$ \\
\hline $\mathbf{2 2 6}$ & 2,5-dimethylphenanthrene & 0.08 & 0.09 & \\
$\mathbf{2 2 7}$ & 1,7-dimethylphenanthrene & & 0.05 & \\
$\mathbf{2 2 9}$ & 2,7-dimethylphenanthrene & 0.28 & 0.27 & \\
$\mathbf{2 3 0}$ & 2,6-dimethylphenanthrene & 0.19 & 0.19 & \\
$\mathbf{2 4 5}$ & retene & 2.73 & 2.70 & 3.86 \\
\hline
\end{tabular}

Table S29. TAPs Detected in LTCT and UP ${ }_{2}$.

\begin{tabular}{llll}
\hline \multirow{2}{*}{ peak } & \multirow{2}{*}{ TAP } & \multicolumn{2}{l}{$\mathrm{RC}$} \\
\cline { 3 - 4 } & & LTCT & $\mathrm{UP}_{2}$ \\
\hline $\mathbf{2 4 2}$ & 2,3,5-trimethylphenanthrene & 0.03 & \\
$\mathbf{2 5 6}$ & 8-isopropyl-1,3-dimethylphenanthrene & 0.14 & 0.16 \\
\hline
\end{tabular}

Table S30. MAs Detected in LTCT and $\mathrm{UP}_{2}$.

\begin{tabular}{|c|c|c|c|c|c|c|c|}
\hline \multirow{2}{*}{ peak } & \multirow{2}{*}{ MA } & $\mathrm{RC}$ & & \multirow{2}{*}{ Peak } & \multirow{2}{*}{ MA } & \multicolumn{2}{|l|}{$\mathrm{RC}$} \\
\hline & & LTCT & $\mathrm{UP}_{2}$ & & & LTCT & $\mathrm{UP}_{2}$ \\
\hline 214 & 1-methylanthracene & 0.77 & 0.62 & 216 & 2-methylanthracene & 0.33 & 0.33 \\
\hline
\end{tabular}

Table S31. DMAs Detected in LTCT and $\mathrm{UP}_{2}$.

\begin{tabular}{|c|c|c|c|c|c|c|}
\hline \multirow{2}{*}{ peak } & \multirow{2}{*}{ DMA } & \multicolumn{2}{|l|}{$\mathrm{RC}$} & \multirow[b]{2}{*}{ peak } & \multirow{2}{*}{ DMA } & \multirow{2}{*}{$\mathrm{RC}$ in $\mathrm{UP}_{2}$} \\
\hline & & LTCT & $\mathrm{UP}_{2}$ & & & \\
\hline 224 & 9,10-dimethylanthracene & 0.08 & & 228 & 1,5-dimethylanthracene & 0.05 \\
\hline 225 & 1,4-dimethylanthracene & 0.09 & 0.08 & & & \\
\hline
\end{tabular}

Table S32. DMDHNs Detected in LTCT and $\mathrm{UP}_{2}$.

\begin{tabular}{|c|c|c|c|c|c|c|c|}
\hline \multirow{2}{*}{ peak } & \multirow{2}{*}{ DMDHN } & \multicolumn{2}{|l|}{$\mathrm{RC}$} & \multirow{2}{*}{ peak } & \multirow{2}{*}{ DMDHN } & \multicolumn{2}{|l|}{$\mathrm{RC}$} \\
\hline & & LTCT & $\mathrm{UP}_{2}$ & & & LTCT & $\mathrm{UP}_{2}$ \\
\hline 61 & 1,4-DMDHN & 0.05 & 0.05 & 80 & 5,8-DMDHN & 0.21 & 0.07 \\
\hline
\end{tabular}

Table S33. TMDHNs Detected in LTCT and HCUP ${ }_{2}$.

\begin{tabular}{lllllll}
\hline \multirow{2}{*}{ peak } & \multirow{2}{*}{ TMDHN } & \multirow{2}{*}{ RC in LTCT } & peak & \multirow{2}{*}{ TMDHN } & \multicolumn{2}{l}{ RC } \\
\cline { 5 - 7 } & & & & & LTCT & HCUP $_{2}$ \\
\hline 118 & $1,1,6-T M D H N$ & 0.04 & $\mathbf{1 7 8}$ & $2,5,8-T M D H N$ & 0.42 & 0.60 \\
\hline
\end{tabular}

Table S34. MDHAs Detected in LTCT.

\begin{tabular}{llllll}
\hline peak & MDHA & RC & peak & MDHA & RC \\
\hline 197 & 1-methyl-9,10-dihydrophenanthrene & 0.06 & $\mathbf{2 0 2}$ & 2-methyl-9,10-dihydroanthracene & 0.09 \\
\hline
\end{tabular}


Table S35. OHAs Detected in LTCT and UP $\mathbf{P}_{2}$.

\begin{tabular}{|c|c|c|c|c|c|c|}
\hline \multirow{2}{*}{ peak } & \multirow{2}{*}{ OHA } & \multicolumn{2}{|l|}{$\mathrm{RC}$} & \multirow[b]{2}{*}{ peak } & \multirow{2}{*}{$\mathrm{OHA}$} & \multirow{2}{*}{$\mathrm{RC}$ in $\mathrm{UP}_{2}$} \\
\hline & & LTCT & $\mathrm{UP}_{2}$ & & & \\
\hline 123 & acenaphthene & 0.63 & 0.34 & 252 & 5,12-dihydrotetracene & 0.04 \\
\hline \multicolumn{7}{|c|}{ Table S36. AAs ${ }^{I}$ Detected in $\mathbf{U P}_{2}$. } \\
\hline peak & $\mathrm{AA}^{\mathrm{I}}$ & & $\mathrm{RC}$ & & $\mathrm{AA}^{\mathrm{I}}$ & $\mathrm{RC}$ \\
\hline 64 & 2-mesitylpro & & 0.15 & 1 & 2-allylnaphthalene & 0.30 \\
\hline
\end{tabular}

Table S37. OSAs Detected in LTCT, $\mathbf{L P}_{2}$ and $\mathbf{U P}_{2}$.

\begin{tabular}{lllll}
\multirow{2}{*}{ peak } & \multirow{2}{*}{ OSA } & $\mathrm{RC}$ & & \\
\cline { 3 - 5 } & & $\mathrm{LTCT}$ & $\mathrm{UP}_{2}$ & $\mathrm{HCUP}_{2}$ \\
\hline $\mathbf{1 7 2}$ & azulene & 0.33 & 0.37 & 0.27 \\
$\mathbf{2 1 7}$ & 1,1-di( $p$-tolyl)ethene & 0.10 & & \\
$\mathbf{2 3 7}$ & 5-methylenedibenzo[ $a, d][7]$ annulene & 0.20 & & \\
$\mathbf{2 5 0}$ & 1-methylpyrene & 0.15 & 0.12 & \\
\hline
\end{tabular}

Table S38. NSAs ${ }^{\mathrm{II}}$ Detected in LTCT and $\mathrm{LP}_{2}$.

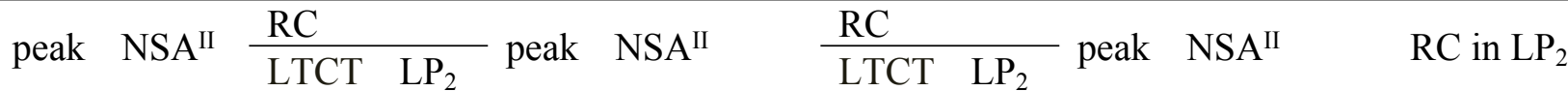

$\begin{array}{lllllllllll}2 & \text { phenol } & 0.27 & 2.54 & \mathbf{1 2 7} & \text { naphth-2-ol } & 0.70 & 1.41 & \mathbf{1 2 8} & \text { naphth-1-ol } & 3.29\end{array}$

Table S39. Cresols Detected in LTCT, $\mathrm{LP}_{2}$ and $\mathrm{UP}_{2}$.

\begin{tabular}{lllllllll}
\hline \multirow{2}{*}{ peak } & \multirow{2}{*}{ cresol } & \multicolumn{2}{l}{ RC } & & & \multirow{2}{*}{ peak } & cresol & \multicolumn{2}{l}{ RC } \\
\cline { 3 - 5 } & & LTCT & $\mathrm{LP}_{2}$ & $\mathrm{UP}_{2}$ & & LTCT & $\mathrm{UP}_{2}$ \\
\hline $\mathbf{6}$ & $o$-cresol & 1.59 & 4.08 & & $\mathbf{1 1}$ & $m$-cresol & 0.05 & 0.09 \\
$\mathbf{9}$ & $p$-cresol & 6.15 & 21.20 & 0.49 & & & & \\
\hline
\end{tabular}

Table S40. EPs Detected in LTCT, $\mathrm{LP}_{2}$, and $\mathrm{UP}_{2}$.

\begin{tabular}{|c|c|c|c|c|c|c|c|c|}
\hline \multirow{2}{*}{ peak } & \multirow{2}{*}{ EP } & \multicolumn{3}{|l|}{$\mathrm{RC}$} & \multirow{2}{*}{ peak } & \multirow{2}{*}{ EP } & \multicolumn{2}{|l|}{$\mathrm{RC}$} \\
\hline & & LTCT & $\mathrm{LP}_{2}$ & $\mathrm{UP}_{2}$ & & & LTCT & $\mathrm{LP}_{2}$ \\
\hline 14 & 2-EP & 0.45 & 1.19 & 0.11 & 21 & 3-EP & 0.13 & 2.10 \\
\hline
\end{tabular}

Table S41. PPs Detected in LTCT and LP .

\begin{tabular}{|c|c|c|c|c|c|c|c|}
\hline \multirow{2}{*}{ peak } & \multirow{2}{*}{ PP } & \multicolumn{2}{|l|}{$\mathrm{RC}$} & \multirow{2}{*}{ peak } & \multirow{2}{*}{ PP } & \multicolumn{2}{|l|}{$\mathrm{RC}$} \\
\hline & & LTCT & $\mathrm{LP}_{2}$ & & & LTCT & $\mathrm{LP}_{2}$ \\
\hline 34 & 2-PP & 0.28 & 0.26 & 36 & 3-PP & 0.79 & 2.33 \\
\hline
\end{tabular}

Table S42. Xylenols Detected in LTCT, LP $_{2}$ and $\mathrm{UP}_{2}$.

\begin{tabular}{|c|c|c|c|c|c|c|c|c|c|}
\hline \multirow{2}{*}{ peak } & \multirow{2}{*}{ xylenol } & \multicolumn{3}{|l|}{$\mathrm{RC}$} & \multirow{2}{*}{ peak } & \multirow{2}{*}{ xylenol } & \multicolumn{3}{|l|}{$\mathrm{RC}$} \\
\hline & & LTCT & $\mathrm{LP}_{2}$ & $\mathrm{UP}_{2}$ & & & LTCT & $\mathrm{LP}_{2}$ & $\mathrm{UP}_{2}$ \\
\hline 13 & 2,6-xylenol & 0.31 & 0.46 & 0.06 & 22 & 3,5-xylenol & 2.60 & 8.92 & 0.54 \\
\hline 16 & 2,4-xylenol & 4.49 & 3.27 & 0.14 & 23 & 2,5-xylenol & 0.70 & 1.58 & 0.17 \\
\hline 17 & 2,3-xylenol & 0.13 & 0.46 & & 26 & 3,4-xylenol & & 4.04 & \\
\hline
\end{tabular}

Table S43. ECs Detected in LTCT and LP.

\begin{tabular}{llllllll}
\hline \multirow{2}{*}{ peak } & \multirow{2}{*}{ EC } & \multicolumn{2}{l}{$\mathrm{RC}$} & \multirow{2}{*}{ eak } & EC & \multicolumn{2}{l}{$\mathrm{RC}$} \\
\cline { 3 - 7 } & & $\mathrm{LTCT}$ & $\mathrm{LP}_{2}$ & & & LTCT & $\mathrm{LP}_{2}$ \\
\hline $\mathbf{2 4}$ & 2-ethyl-4-cresol & 0.26 & 0.26 & $\mathbf{4 7}$ & 4-ethyl-2-cresol & 1.64 & 6.84 \\
$\mathbf{3 8}$ & 2-ethyl-6-cresol & 0.17 & 0.69 & $\mathbf{4 8}$ & 3-ethyl-5-cresol & 0.13 & 0.11 \\
$\mathbf{4 2}$ & 2-ethyl-4-cresol & 1.21 & 3.46 & $\mathbf{5 1}$ & 4-ethyl-3-cresol & & 0.10 \\
$\mathbf{4 4}$ & 2-ethyl-5-cresol & 0.42 & 1.44 & & & & \\
\hline
\end{tabular}


Table S44. PCs Detected in LTCT and $\mathrm{LP}_{2}$.

\begin{tabular}{|c|c|c|c|c|c|c|c|}
\hline \multirow{2}{*}{ peak } & \multirow{2}{*}{$\mathrm{PC}$} & \multicolumn{2}{|l|}{$\mathrm{RC}$} & \multirow{2}{*}{ peak } & \multirow{2}{*}{$\mathrm{PC}$} & \multicolumn{2}{|l|}{$\mathrm{RC}$} \\
\hline & & LTCT & $\mathrm{LP}_{2}$ & & & LTCT & $\mathrm{LP}_{2}$ \\
\hline 63 & 6-propyl-2-cresol & & 0.13 & 72 & 2-propyl-4-cresol & 0.08 & 0.39 \\
\hline 70 & 4-propyl-2-cresol & 0.34 & 0.70 & & & & \\
\hline
\end{tabular}

Table S45. IPCs Detected in LTCT and $\mathrm{LP}_{2}$.

\begin{tabular}{lllllll}
\hline \multirow{2}{*}{ peak } & \multirow{2}{*}{ IPC } & \multicolumn{2}{l}{ RC } & \multirow{2}{*}{ peak } & IPC & \multirow{2}{*}{ RC in $\mathrm{LP}_{2}$} \\
\cline { 3 - 5 } & & LTCT & $\mathrm{LP}_{2}$ & & & \\
\hline $\mathbf{5 4}$ & 4-isopropyl-3-cresol & 0.22 & 0.12 & $\mathbf{6 5}$ & 4-isopropyl-3-cresol & 0.48 \\
$\mathbf{5 8}$ & 5-isopropyl-2-cresol & & 0.71 & $\mathbf{7 8}$ & thymol & 0.10 \\
\hline
\end{tabular}

Table S46. DEPs Detected in $\mathbf{L P}_{2}$.

\begin{tabular}{llllll}
\hline peak & DEP & RC & peak & DEP & RC \\
\hline $\mathbf{6 0}$ & 2,5 -DEP & 0.10 & $\mathbf{9 5}$ & 2,5 -DEP & 0.12 \\
\hline
\end{tabular}

Table S47. TMPs Detected in LTCT, $\mathrm{LP}_{2}$ and $\mathrm{UP}_{2}$.

\begin{tabular}{|c|c|c|c|c|c|c|c|}
\hline \multirow{2}{*}{ peak } & \multirow{2}{*}{ TMP } & \multicolumn{3}{|l|}{$\mathrm{RC}$} & \multirow{2}{*}{ peak } & \multirow{2}{*}{ TMP } & \multirow{2}{*}{$\mathrm{RC}$ in $\mathrm{LP}_{2}$} \\
\hline & & LTCT & $\mathrm{LP}_{2}$ & $\mathrm{UP}_{2}$ & & & \\
\hline 31 & mesitol & 0.49 & 0.81 & 0.09 & 53 & 3,4,6-TMP & 0.45 \\
\hline 49 & 2,3,6-TMP & 0.44 & 1.39 & & 66 & pseudocumenol & 0.55 \\
\hline 50 & 3,4,5-TMP & 0.51 & 1.34 & & & & \\
\hline
\end{tabular}

Table S48. OASPs Detected in LTCT and $L P_{2}$.

\begin{tabular}{lllllll}
\multirow{2}{*}{ peak } & \multirow{2}{*}{ OASP } & $\mathrm{RC}$ & & \multirow{2}{*}{ peak } & OASP & \multirow{2}{*}{ RC in $\mathrm{LP}_{2}$} \\
\cline { 3 - 5 } & & $\mathrm{LTCT}$ & $\mathrm{LP}_{2}$ & & & \multirow{2}{*}{0.94} \\
$\mathbf{8 3}$ & durenol & 0.76 & 0.29 & $\mathbf{1 2 4}$ & 2,5-di-tert-butylphenol \\
$\mathbf{8 8}$ & 2-ethyl-5-propylphenol & & 0.22 & & & \\
\hline
\end{tabular}

Table S49. APs Detected in $\mathbf{L P}_{2}$.

\begin{tabular}{llllll}
\hline peak & AP & RC & peak & AP & RC \\
\hline $\mathbf{6 8}$ & 4-allylphenol & 2.77 & $\mathbf{1 0 2}$ & 4-prenylphenol & 0.16 \\
\hline
\end{tabular}

Table S50. ACs Detected in LTCT and $\mathbf{L P}_{2}$.

\begin{tabular}{|c|c|c|c|c|c|c|}
\hline \multirow{2}{*}{ peak } & \multirow{2}{*}{$\mathrm{AC}$} & \multicolumn{2}{|l|}{$\mathrm{RC}$} & \multirow{2}{*}{ peak } & \multirow{2}{*}{$\mathrm{AC}$} & \multirow{2}{*}{$\mathrm{RC}$ in $\mathrm{LP}_{2}$} \\
\hline & & LTCT & $\mathrm{LP}_{2}$ & & & \\
\hline $\begin{array}{l}82 \\
100\end{array}$ & $\begin{array}{l}\text { 2-allyl-6-cresol } \\
\text { 2-allyl-4-cresol }\end{array}$ & 0.21 & $\begin{array}{l}0.17 \\
0.77\end{array}$ & 137 & 2-(pent-3-en-2-yl)-4-cresol & 0.45 \\
\hline
\end{tabular}

Table S51. NSIs Detected in LTCT and $L P_{2}$.

\begin{tabular}{lllllllll}
\hline peak & NSI & RC in $\mathrm{LP}_{2}$ & peak & NSI & RC in LTCT & peak & NSI & RC in $\mathrm{LP}_{2}$ \\
\hline $\mathbf{7 4}$ & $\begin{array}{l}\text { indan-5-o } \\
1\end{array}$ & 0.12 & $\mathbf{7 6}$ & $\begin{array}{l}\text { indan-1-o } \\
1\end{array}$ & 0.59 & $\mathbf{7 7}$ & $\begin{array}{l}\text { indan-4-o } \\
1\end{array}$ & 5.19 \\
\hline
\end{tabular}

Table S52. MIs Detected in LTCT and $\mathrm{LP}_{2}$.

\begin{tabular}{llllllll}
\hline \multirow{2}{*}{ peak } & \multirow{2}{*}{$\mathrm{MI}$} & \multicolumn{2}{l}{$\mathrm{RC}$} & \multicolumn{2}{l}{ peak } & $\mathrm{MI}$ & $\mathrm{RC}$ \\
\cline { 3 - 7 } & & $\mathrm{LTCT}$ & $\mathrm{LP}_{2}$ & & & $\mathrm{LTCT}$ & $\mathrm{LP}_{2}$ \\
\hline $\mathbf{9 1}$ & 5-methylindan-4-ol & & 0.62 & $\mathbf{1 0 6}$ & 2-methylindan-1-ol & 0.10 & 0.98 \\
$\mathbf{1 0 1}$ & 7-methylindan-4-ol & 0.19 & 0.76 & $\mathbf{1 1 0}$ & 7-methylindan-5-ol & & 1.86 \\
$\mathbf{1 0 4}$ & 6-methylindan-4-ol & & 1.08 & & & & \\
\hline
\end{tabular}

Table S53. DMIs ${ }^{\mathrm{II}}$ Detected in LTCT and $L P_{2}$.

\begin{tabular}{lllllllll}
\hline peak & $\mathrm{DMI}^{\mathrm{II}}$ & $\mathrm{RC}$ in $\mathrm{LP}_{2}$ & peak & $\mathrm{DMI}^{\mathrm{II}}$ & $\mathrm{RC}$ in LTCT & peak & $\mathrm{DMI}^{\mathrm{II}}$ & $\mathrm{RC}$ in LTCT \\
\hline $\mathbf{1 0 8}$ & $3,3-\mathrm{DMI}^{\mathrm{II}}$ & 0.12 & $\mathbf{1 1 6}$ & $2,2-\mathrm{DMI}^{\mathrm{II}}$ & 0.35 & $\mathbf{1 3 4}$ & $2,3-\mathrm{DMI}^{\mathrm{II}}$ & 0.30 \\
\hline
\end{tabular}


Table S54. MNs ${ }^{\mathrm{II}}$ Detected in LTCT, $\mathrm{LP}_{2}$ and $\mathrm{UP}_{2}$.

\begin{tabular}{|c|c|c|c|c|c|c|c|c|c|c|}
\hline \multirow{2}{*}{ peak } & \multirow{2}{*}{$\mathrm{MN}^{\mathrm{II}}$} & \multicolumn{3}{|l|}{$\mathrm{RC}$} & \multirow{2}{*}{ peak } & \multirow{2}{*}{$\mathrm{MN}^{\mathrm{II}}$} & \multirow{2}{*}{$\mathrm{RC}$ in $\mathrm{LP}_{2}$} & \multirow{2}{*}{ peak } & \multirow{2}{*}{$\mathrm{MN}^{\mathrm{II}}$} & \multirow[b]{2}{*}{$\mathrm{RC}$ in $\mathrm{LP}_{2}$} \\
\hline & & LTCT & $\mathrm{LP}_{2}$ & $\mathrm{UP}_{2}$ & & & & & & \\
\hline 152 & 2-MNII & 0.07 & 1.67 & & 156 & 5-MN & 0.12 & 161 & 3-MNII & 1.17 \\
\hline 153 & 7-MN & 0.25 & 0.49 & & 158 & 8-MN ${ }^{I I}$ & 0.26 & 162 & 6-MN ${ }^{\mathrm{II}}$ & 0.22 \\
\hline 154 & 4-MN ${ }^{\mathrm{II}}$ & 0.28 & 0.82 & 0.18 & & & & & & \\
\hline
\end{tabular}

Table S55. DMNs Detected in LTCT and $L P_{2}$.

\begin{tabular}{lllllll}
\multirow{2}{*}{ peak } & \multirow{2}{*}{$\mathrm{DMN}$} & \multicolumn{2}{l}{$\mathrm{RC}$} & \multirow{2}{*}{ peak } & $\mathrm{DMN}$ & \multirow{2}{*}{$\mathrm{RC}$ in $\mathrm{LP}_{2}$} \\
\cline { 3 - 6 } & & $\mathrm{LTCT}$ & $\mathrm{LP}_{2}$ & & \\
\hline $\mathbf{1 4 3}$ & $2,5-\mathrm{DMN}$ & & 0.18 & $\mathbf{1 5 5}$ & $5,6-\mathrm{DMN}$ & 0.91 \\
$\mathbf{1 5 2}$ & $6,7-\mathrm{DMN}$ & 0.20 & 0.30 & $\mathbf{1 5 9}$ & $5,7-\mathrm{DMN}$ & 0.47 \\
\hline
\end{tabular}

Table S56. MSDBFs Detected in LTCT and $\mathrm{UP}_{2}$.

\begin{tabular}{lllllll}
\hline \multirow{2}{*}{ peak } & \multirow{2}{*}{ MSDBF } & \multicolumn{2}{l}{ RC } & \multirow{2}{*}{ peak } & MSDBF & \multirow{2}{*}{ RC in LTCT } \\
\cline { 3 - 6 } & & LTCT & $\mathrm{UP}_{2}$ & & & \\
\hline $\mathbf{1 5 5}$ & 4-methyldibenzofuran & 1.09 & 1.04 & $\mathbf{1 7 3}$ & 3-methyldibenzofuran & 0.06 \\
$\mathbf{1 6 0}$ & 1-methyldibenzofuran & 0.91 & 0.94 & $\mathbf{1 8 4}$ & 3,4-dimethyldibenzofuran & 0.15 \\
$\mathbf{1 6 3}$ & 2-methyldibenzofuran & 0.18 & 0.16 & & & \\
\hline
\end{tabular}

Table S57. NBFs Detected in LTCT and UP $P_{2}$.

\begin{tabular}{lllllll}
\hline \multirow{2}{*}{ peak } & \multirow{2}{*}{ NBF } & \multicolumn{2}{l}{ RC } & \multirow{2}{*}{ peak } & \multirow{2}{*}{ NBF } & \multirow{2}{*}{ RC in LTCT } \\
\cline { 3 - 6 } & & LTCT & UP $_{2}$ & & & \\
\hline $\mathbf{2 3 6}$ & naphtho[2,3-b]benzofuran & 0.68 & 0.72 & $\mathbf{2 3 8}$ & naphtho[2,1-b]benzofuran & 0.21 \\
\hline
\end{tabular}

Table S58. Alkenones Detected in LTCT and LP $_{2}$.

\begin{tabular}{lllllll}
\multirow{2}{*}{ peak } & \multirow{2}{*}{ alkenone } & \multicolumn{2}{l}{ RC } & \multirow{2}{*}{ peak } & \multirow{2}{*}{ alkenone } & RC in \\
\cline { 3 - 5 } & & LTCT & $\mathrm{LP}_{2}$ & & & LTCT \\
\hline $\mathbf{5}$ & 2,3-dimethylcyclopent-2-enone & 0.20 & 0.38 & $\mathbf{1 0}$ & 1-acetyl-1-cyclohexene & 0.10 \\
$\mathbf{7}$ & 2,3,4-trimethylcyclopent-2-enone & 0.11 & 0.13 & & & \\
\hline
\end{tabular}

Table S59. OOCOCs Detected in LTCT.

\begin{tabular}{llllll}
\hline peak & OOCOC & RC & peak & OOCOC & RC \\
\hline $\mathbf{1 6 4}$ & dibenzo[ $b, d]$ pyran & 0.08 & $\mathbf{1 8 8}$ & dibenzofuran-2-carbaldehyde & 0.43 \\
$\mathbf{1 8 6}$ & fluoren-9-ol & 0.11 & $\mathbf{1 9 0}$ & 5,7-dihydrodibenzo[c,e]osipine & 0.18 \\
\hline
\end{tabular}

Table S60. DAPs Detected in LTCT.

\begin{tabular}{llllll}
\hline peak & DAP & RC & peak & DAP & RC \\
\hline $\mathbf{1}$ & 3,5-dimethylpyridine & 0.09 & $\mathbf{8}$ & 2,6-diethylpyridine & 0.03 \\
\hline
\end{tabular}

Table S61. TMPs Detected in LTCT.

\begin{tabular}{llllll}
\hline peak & TMP & RC & peak & TMP & RC \\
\hline $\mathbf{3}$ & 2,4,6-trimethylpyridine & 0.12 & $\mathbf{4}$ & $2,3,6$-trimethylpyridine & 0.06 \\
\hline
\end{tabular}

Table S62. OSPs Detected in LTCT and UP $P_{2}$.

\begin{tabular}{llllll}
\hline peak & OSP & $\mathrm{RC}_{\text {in } \mathrm{UP}_{2}}$ & peak & $\mathrm{OSP}$ & RC in LTCT \\
\hline $\mathbf{7 5}$ & 2-methyl-5-vinylpyridine & 0.04 & $\mathbf{2 0 4}$ & 3-phenylpyridine & 0.07 \\
\hline
\end{tabular}

Table S63. AA II Detected in LTCT and UP.

\begin{tabular}{|c|c|c|c|c|c|c|}
\hline \multirow{2}{*}{ peak } & \multirow{2}{*}{$\mathrm{AA}^{\mathrm{II}}$} & \multirow{2}{*}{$\mathrm{RC}$ in LTCT } & \multirow{2}{*}{ peak } & \multirow{2}{*}{$\mathrm{AA}^{\mathrm{II}}$} & \multicolumn{2}{|l|}{$\mathrm{RC}$} \\
\hline & & & & & LTCT & $\mathrm{UP}_{2}$ \\
\hline $\begin{array}{l}223 \\
243\end{array}$ & $\begin{array}{l}\text { 9-methylacridine } \\
\text { 2-ethylacridine }\end{array}$ & $\begin{array}{l}0.11 \\
0.06\end{array}$ & 246 & 2-ethylacridine & 0.31 & 0.02 \\
\hline
\end{tabular}

Table S64. DMBIs Detected in $\mathbf{U P}_{2}$.

\begin{tabular}{llllll}
\hline peak & DMBI & RC & peak & DMBI & RC \\
\hline $\mathbf{3 3}$ & 5,6 -dimethylbenzimida & 0.03 & $\mathbf{3 5}$ & 1,2-dimethylbenzimidazole & 0.23 \\
\hline
\end{tabular}


zole

Table S65. AAs ${ }^{\mathrm{III}}$ Detected in LTCT and $\mathrm{UP}_{2}$.

\begin{tabular}{|c|c|c|c|c|c|c|}
\hline \multirow{2}{*}{ peak } & \multirow{2}{*}{$\mathrm{AA}^{\mathrm{III}}$} & $\mathrm{RC}$ & & \multirow{2}{*}{ peak } & \multirow{2}{*}{$\mathrm{AA}^{\mathrm{III}}$} & \multirow{2}{*}{$\mathrm{RC}$ in LTCT } \\
\hline & & LTCT & $\mathrm{UP}_{2}$ & & & \\
\hline 109 & 5-tetralylamine & 0.22 & 0.50 & 159 & 2-methylquinolin-6-amine & 0.08 \\
\hline
\end{tabular}

Table S66. ONCAs Detected in LTCT.

\begin{tabular}{llllll}
\hline peak & ONCA & RC & peak & ONCA & RC \\
\hline $\mathbf{4 6}$ & quinoline & 0.12 & $\mathbf{2 1 8}$ & 3-methylcarbazole & 0.09 \\
$\mathbf{6 7}$ & 2,5-diethylpyrazine & 0.22 & & & \\
\hline
\end{tabular}

Table S67. A SCA Detected in LTCT.

\begin{tabular}{lll}
\hline peak & SCA & RC \\
\hline $\mathbf{1 9 6}$ & thioxanthene & 0.08 \\
\hline
\end{tabular}


$\square \mathrm{LTCT} \square \mathrm{LP}_{2} \nabla \mathrm{UP}_{2} \square \mathrm{HCUP}_{2}$
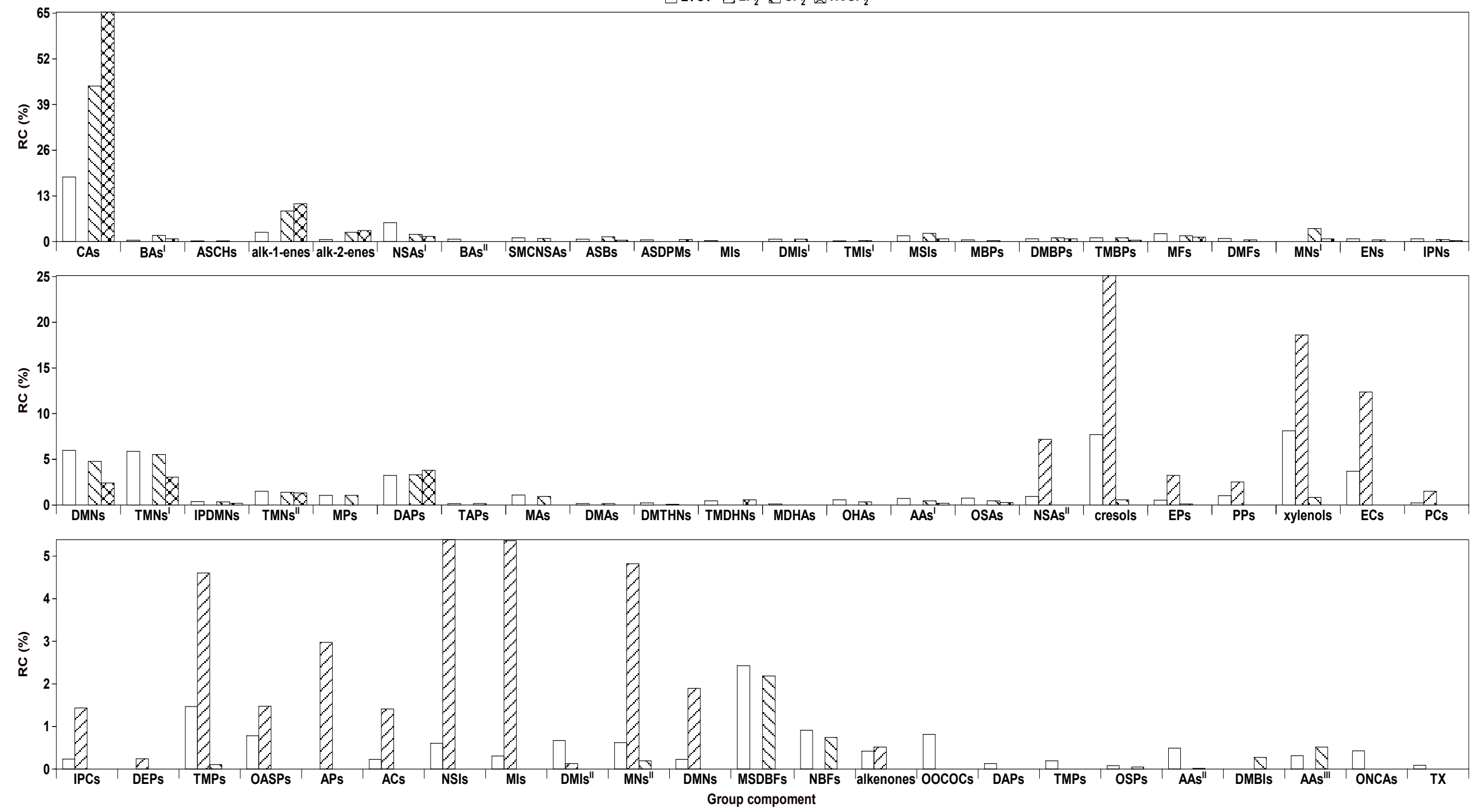

Figure S5. Distribution of group components in the LTCT, $\mathrm{LP}_{2}, \mathrm{UP}_{2}$, and $\mathrm{HCUP}_{2}$. 
Table S68. Classification of the Compounds Detected in the Samples.

\begin{tabular}{|c|c|c|}
\hline full name & nomenclature & Table \\
\hline normal alkanes & NAs & $\mathrm{S} 2$ \\
\hline branched alkanes & $\mathrm{BAs}^{\mathrm{I}}$ & S3 \\
\hline Alkyl-substituted cyclohexanes & ASCHs & S4 \\
\hline alk-1-enes & & S5 \\
\hline alk-2-enes & & S6 \\
\hline non-substituted arenes & NSAs ${ }^{I}$ & S7 \\
\hline biaryls & $\mathrm{BAs}^{\mathrm{II}}$ & S8 \\
\hline single methylene-containing no substituted arenes & SMCNSAs & S9 \\
\hline alkyl-substituted benzenes & ASBs & $\mathrm{S} 10$ \\
\hline alkyl-substituted diphenylmethanes & ASDPMs & $\mathrm{S} 11$ \\
\hline methylindenes & MIs & $\mathrm{S} 12$ \\
\hline dimethylindenes & $\mathrm{DMIs}^{\mathrm{I}}$ & $\mathrm{S} 13$ \\
\hline trimethylindenes & TMIs $^{I}$ & $\mathrm{~S} 14$ \\
\hline methyl-substituted indanes & MSIs & $\mathrm{S} 15$ \\
\hline methylbiphenyls & MBPs & S16 \\
\hline dimethylbiphenyls & DMBPs & S17 \\
\hline tetramethylbiphenyls & TMBPs & $\mathrm{S} 18$ \\
\hline methylfluorenes & MFs & S19 \\
\hline dimethylfluorenes & DMFs & $\mathrm{S} 20$ \\
\hline methylnaphthalenes & $\mathrm{MNs}^{\mathrm{I}}$ & $\mathrm{S} 21$ \\
\hline ethylnaphthalenes & ENs & $\mathrm{S} 22$ \\
\hline isopropylnaphthalenes & IPNs & $\mathrm{S} 23$ \\
\hline dialkylnaphthalenes & DANs & $\mathrm{S} 24$ \\
\hline trimethylnaphthalenes & $\mathrm{TMNs}^{\mathrm{I}}$ & $\mathrm{S} 25$ \\
\hline isopropyldimethylnaphthalenes & IPDMNs & S26 \\
\hline tetramethylnaphthalenes & TMNs ${ }^{I I}$ & S27 \\
\hline methylphenanthrenes & MPs & $\mathrm{S} 28$ \\
\hline dialkylphenanthrenes & DAPs & $\mathrm{S} 29$ \\
\hline trialkylphenanthrenes & TAPs & $\mathrm{S} 30$ \\
\hline methylanthracenes & MAs & S31 \\
\hline dimethylanthracenes & DMAs & S32 \\
\hline dimethyltetrahydronaphthalenes & DMTHNs & S33 \\
\hline trimethyldihydronaphthalene & TMDHNs & S34 \\
\hline methyl-9,10-dihydroarenes & MDHAs & $\mathrm{S} 35$ \\
\hline other hydroarenes & OHAs & $\mathrm{S} 36$ \\
\hline alkenylarenes & $\mathrm{AAs}^{\mathrm{I}}$ & $\mathrm{S} 37$ \\
\hline other-substituted arenes & OSAs & S38 \\
\hline non-substituted arenols & $\mathrm{NSAs}^{\mathrm{II}}$ & $\mathrm{S} 39$ \\
\hline cresols & & $\mathrm{S} 40$ \\
\hline ethylphenols & EPs & S41 \\
\hline propylphenols & PPs & S42 \\
\hline xylenols & & $\mathrm{S} 43$ \\
\hline
\end{tabular}


Table S68. Classification of the Compounds Detected in the Samples (Continued).

\begin{tabular}{lll}
\hline full name & nomenclature & Table \\
\hline ethylcresols & $\mathrm{ECs}$ & $\mathrm{S} 44$ \\
propylcresols & $\mathrm{PCs}$ & $\mathrm{S} 45$ \\
isopropylcresols & $\mathrm{IPCs}$ & $\mathrm{S} 46$ \\
diethylphenols & $\mathrm{DEPs}$ & $\mathrm{S} 47$ \\
trimethylphenols & $\mathrm{TMPs}$ & $\mathrm{S} 48$ \\
Other alkyl-substituted phenols & $\mathrm{OASP}$ & $\mathrm{S} 49$ \\
alkenylphenols & $\mathrm{APs}$ & $\mathrm{S} 50$ \\
alkylcresols & $\mathrm{ACs}$ & $\mathrm{S} 51$ \\
non-substituted indanols & $\mathrm{NSIs}$ & $\mathrm{S} 52$ \\
methylindanols & $\mathrm{MIs}$ & $\mathrm{S} 53$ \\
dimethylindanols & $\mathrm{DMIs}$ & $\mathrm{SI} 54$ \\
methylnaphth-1-ols & $\mathrm{MNs}{ }^{\mathrm{II}}$ & $\mathrm{S} 55$ \\
dimethylnaphth-1-ols & $\mathrm{DMNs}$ & $\mathrm{S} 56$ \\
methyl-substituted dibenzofurans & $\mathrm{MSDBFs}$ & $\mathrm{S} 57$ \\
naphthobenzofurans & $\mathrm{NBFs}$ & $\mathrm{S} 58$ \\
alkenones & & $\mathrm{S} 59$ \\
other oxygen-containing organic compounds & $\mathrm{OOCOCs}$ & $\mathrm{S} 60$ \\
dialkylpyridines & $\mathrm{DAPs}$ & $\mathrm{S} 61$ \\
trimethylpyridines & $\mathrm{TMPs}$ & $\mathrm{S} 62$ \\
other substituted pyridines & OSPs & $\mathrm{S} 63$ \\
alkylacridines & $\mathrm{AAs}$ & $\mathrm{S}$ \\
dimethylbenzimidazoles & $\mathrm{DMBIs}$ \\
aromatic amines & $\mathrm{AAs}{ }^{\mathrm{III}}$ & $\mathrm{S} 65$ \\
other nitrogen-containing aromatics & $\mathrm{ONCAs}$ & $\mathrm{S} 66$ \\
thioxanthene & $\mathrm{TX}$ & $\mathrm{S} 67$ \\
\hline & & $\mathrm{S} 68$ \\
\hline
\end{tabular}



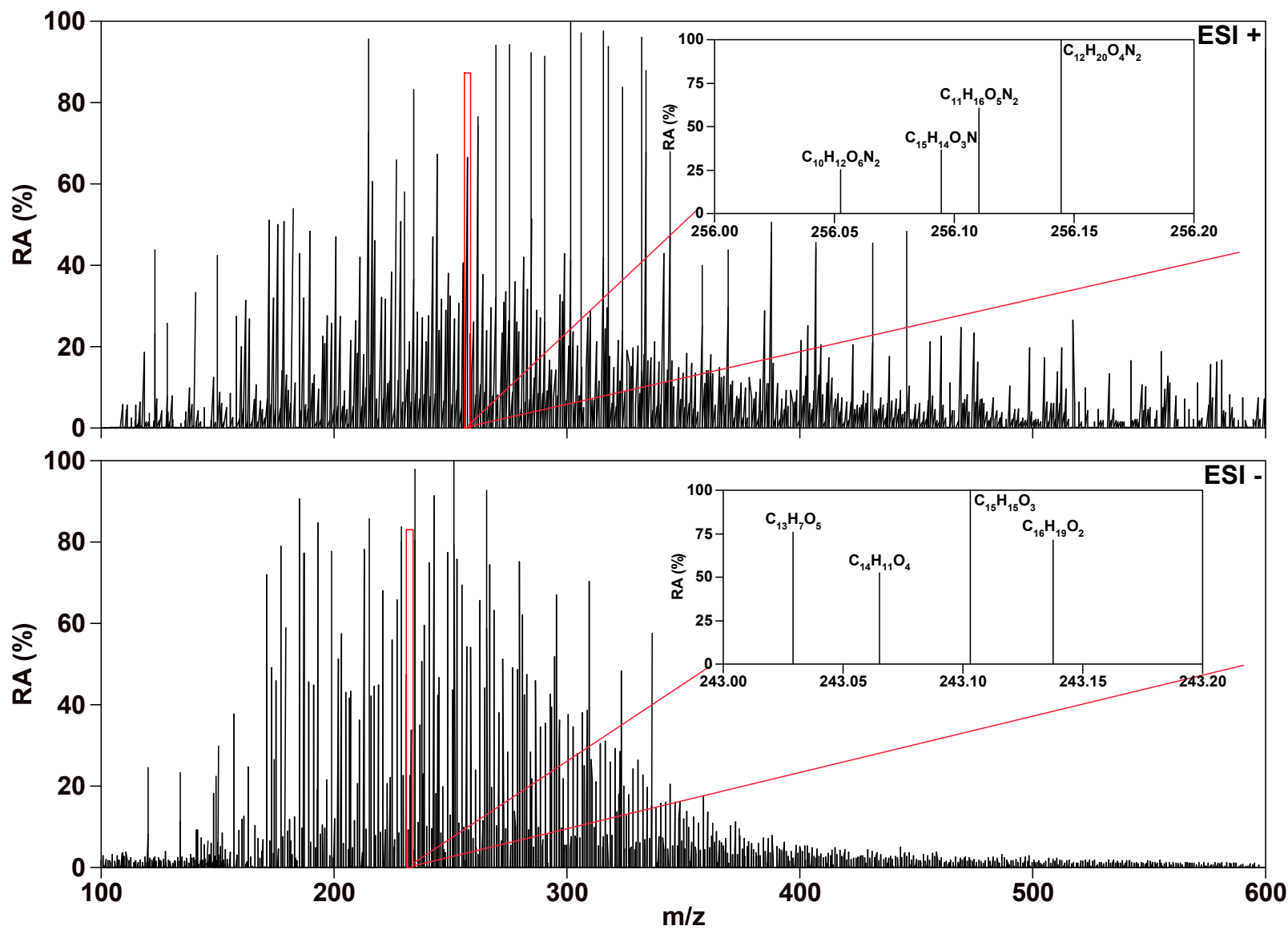

Figure S6. Broadband mass spectra from the analysis of the LTCT with PIM and NIM QEOTMS. 

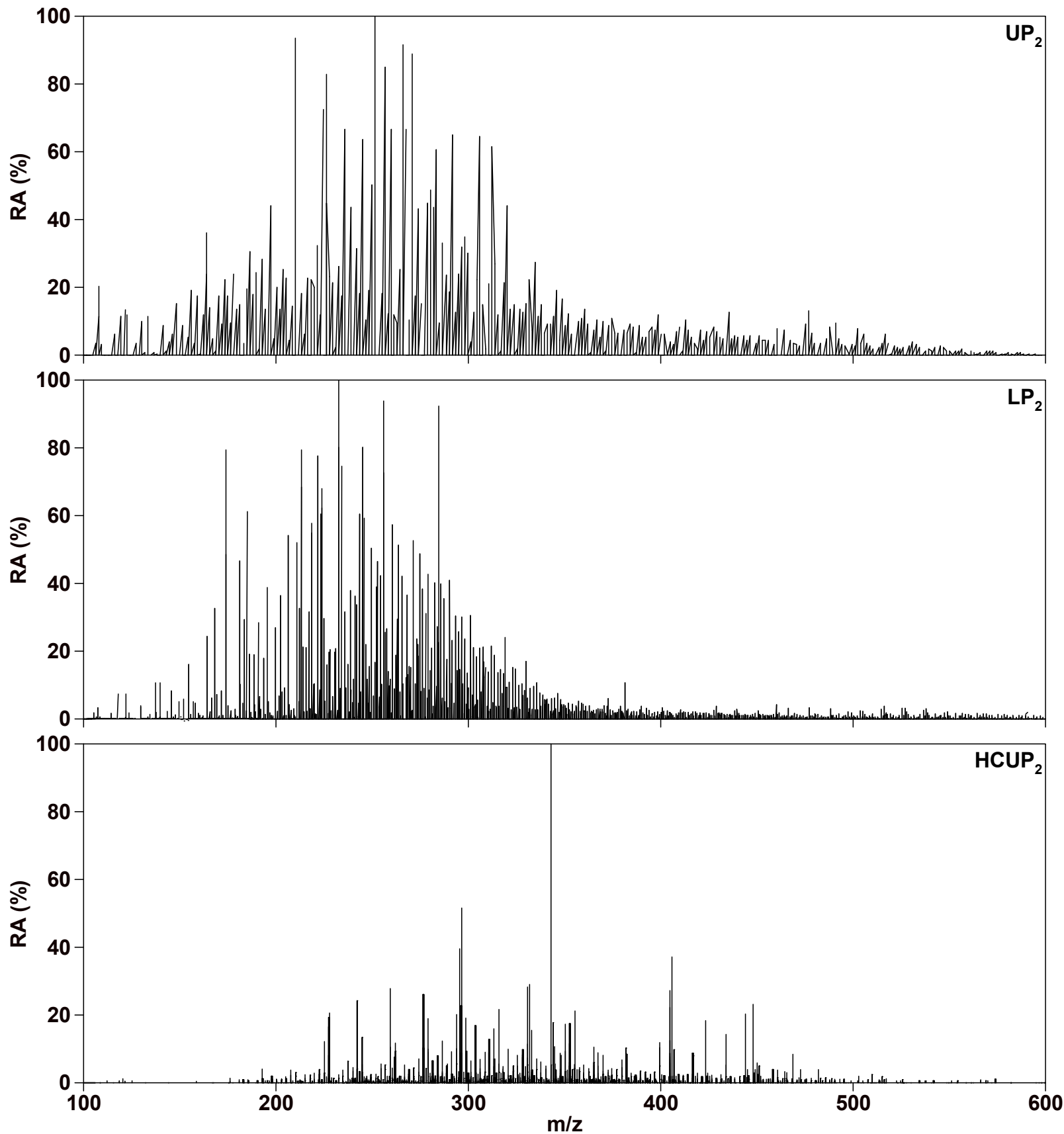

Figure S7. Broadband mass spectra from the analysis of $\mathrm{UP}_{2}, \mathrm{LP}_{2}$ and $\mathrm{HCUP}_{2}$ with NIM QEOTMS. 

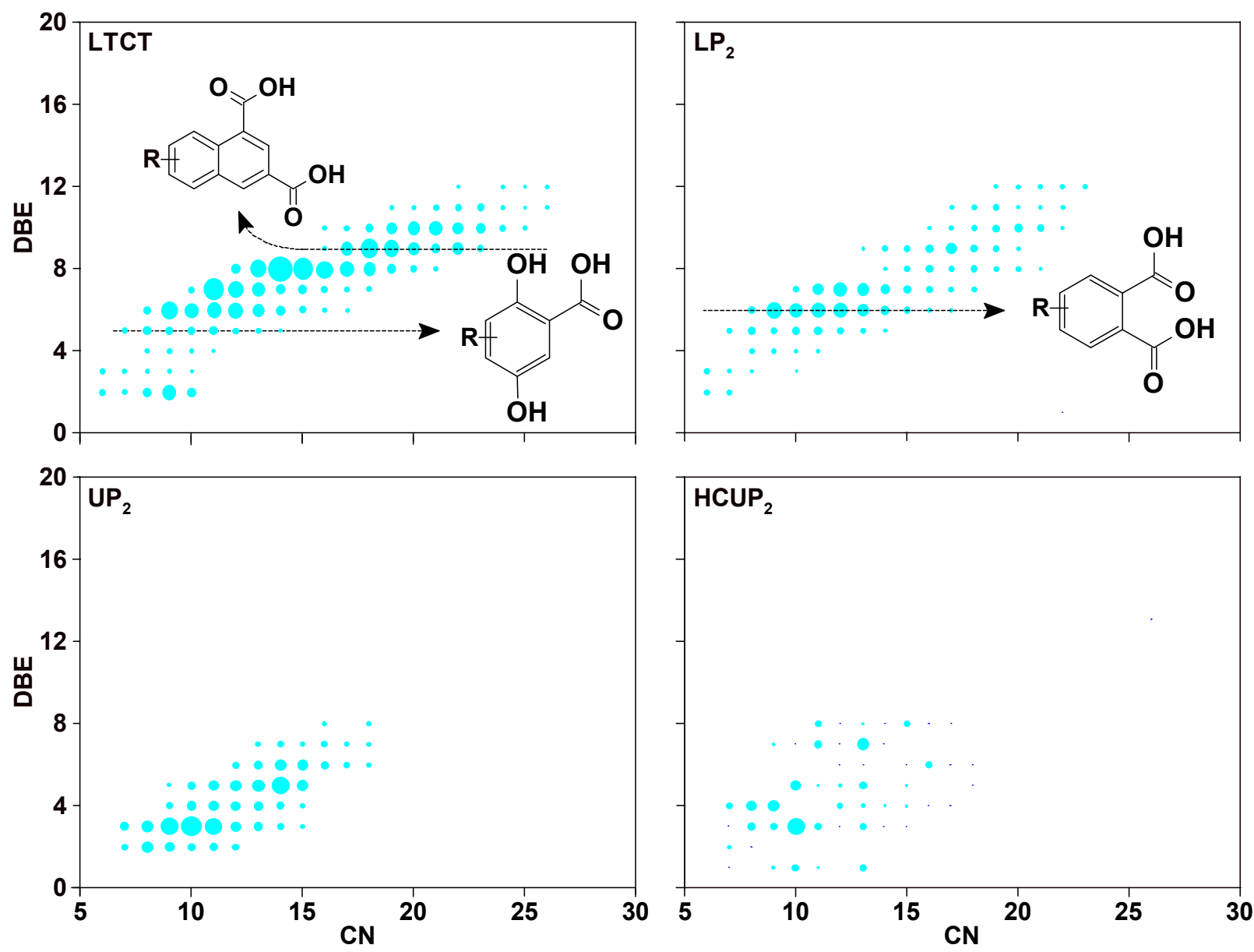

Figure S8. DBE versus $\mathrm{CN}$ for $\mathrm{O}_{4}$ class species analyzed in NIM QEOTMS with supposed structures. 

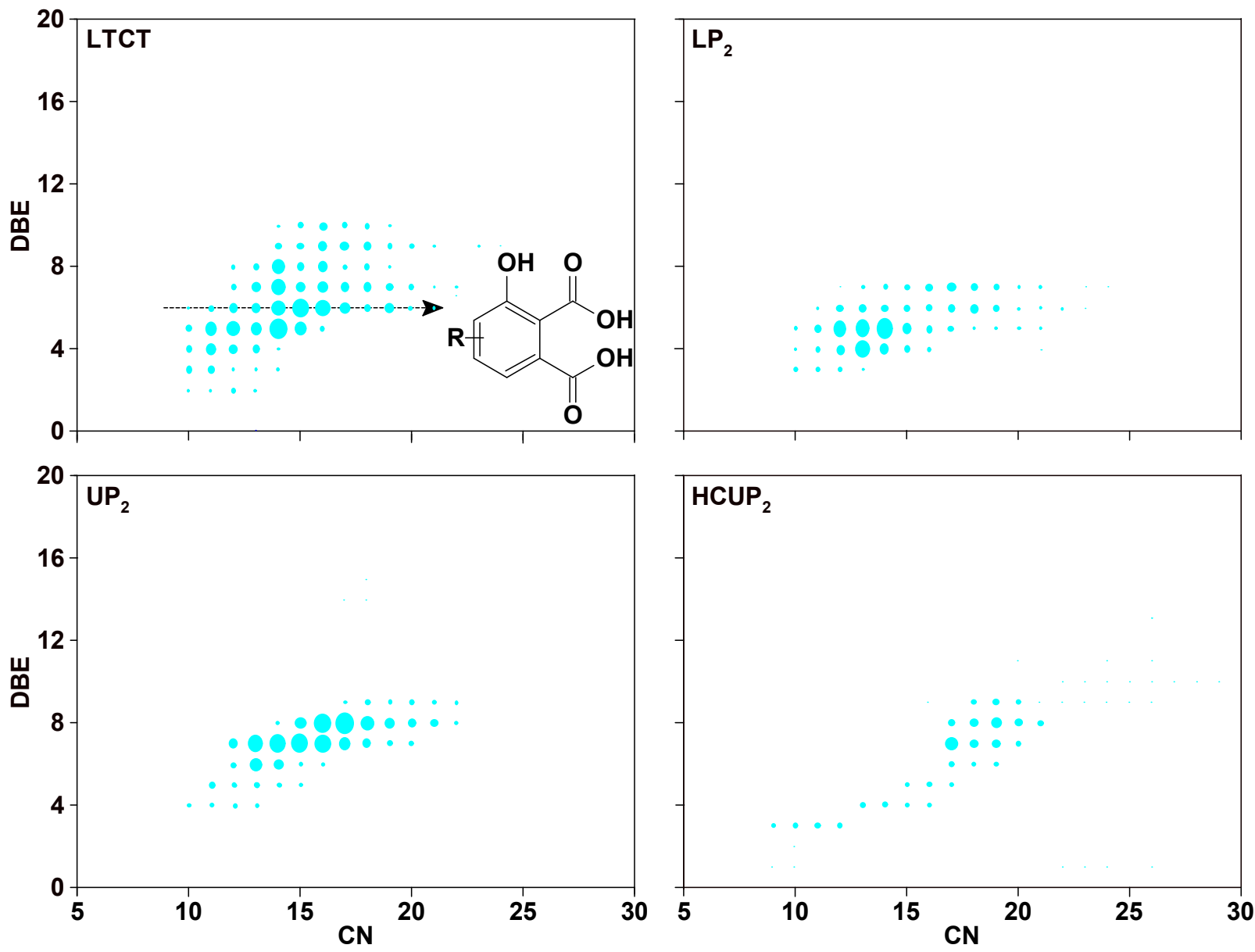

Figure S9. DBE versus $\mathrm{CN}$ for $O_{5}$ class species analyzed in NIM QEOTMS with supposed structures. 

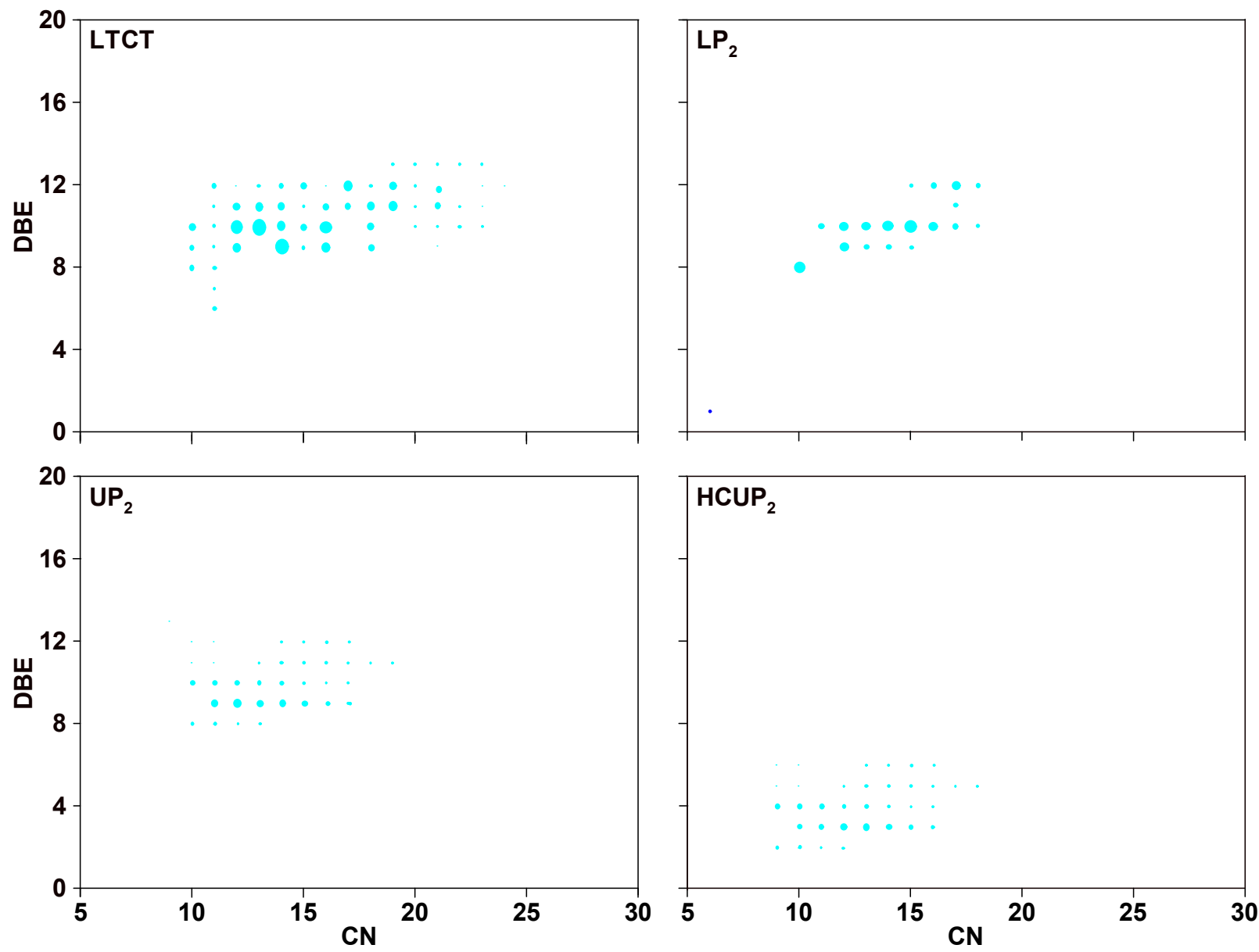

Figure S10. DBE versus $\mathrm{CN}$ for $O_{6}$ class species analyzed in NIM QEOTMS. 